\title{
Neural Correlates of Predictive and Postdictive Switching Mechanisms for Internal Models
}

\author{
Hiroshi Imamizu ${ }^{1,2}$ and Mitsuo Kawato ${ }^{1}$ \\ ${ }^{1}$ Advanced Telecommunications Research Institute International, Computational Neuroscience Laboratories, and ${ }^{2}$ National Institute of Information and \\ Communications Technology, Kyoto 619-0288, Japan
}

\begin{abstract}
Switching of sensorimotor tasks may be classified into predictive switching based on contextual information and postdictive switching based on the error between sensorimotor feedback and predictions. We used functional neuroimaging to study the brain regions involved in each type of switching of internal models for visuomotor rotations (clockwise and counterclockwise rotations of visual feedback). The color of a cue presented before movement initiation corresponded to direction of rotation of the feedback in an instructed condition, but not in a noninstructed condition. Switching-related activity was identified as activity that transiently increased after the direction of rotation was changed. The switching-related activity in cue periods in the instructed condition, when a predictive switch is possible, was observed in the superior parietal lobule (SPL). However, the switching-related activity in feedback periods in the noninstructed condition, when prediction error is crucial for the postdictive switch, was observed in the inferior parietal lobule (IPL) and prefrontal cortex. The functional influence of the SPL on the lateral cerebellum, namely, a possible neural correlate for internal models, increased in the instructed condition, but the influence of the IPL on the cerebellum was increased in the noninstructed condition. We observed a rapid activity increase in the instructed condition and a gradual activity increase in the noninstructed condition mainly in the lateral occipitotemporal cortices (LOTC) and supplementary motor cortex (SMA). These results are consistent with separate mechanisms for predictive and postdictive switches and suggest that the LOTC and SMA receive output signals from appropriate internal models.
\end{abstract}

Key words: task switching; internal models; visuomotor rotation; contextual information; sensorimotor feedback; functional magnetic resonance imaging

\section{Introduction}

Skilled manipulation of objects, including our own bodies, relies on the brain learning to control the object and predict the consequences of this control. Neural mechanisms that mimic the input-output properties of objects support prediction and control; these are termed internal models (Kawato et al., 1987; Wolpert et al., 1995). Considerable evidence from behavioral, neurophysiological, and functional imaging studies indicates that the CNS maintains a number of internal models for different objects and environments in a modular manner (Ghahramani and Wolpert, 1997; Flanagan et al., 1999; Krakauer et al., 1999; Gribble and Scott, 2002; Imamizu et al., 2003; Yamamoto et al., 2007). Behavioral studies have shown that humans can switch internal models based on contextual information. For example, an auditory tone cue can induce context-dependent adaptation to prismatic displacement (Kravitz and Yaffe, 1972). Contextual cues, such as color, can contribute to simultaneous learning and switching between internal models representing different dynamic environments (Wada et al., 2003; Osu et al., 2004). However, little is

Received March 13, 2008; revised July 26, 2008; accepted Sept. 10, 2008

We thank K. Kurimoto for technical support and Drs. H. Tanaka and T. Kouchiyama for helpful comments on this manuscript.

Correspondence should be addressed to Hiroshi Imamizu, National Institute of Information and Communications Technology, 2-2-2 Hikaridai, Keihanna Science City, Kyoto 619-0288, Japan. E-mail: imamizu@gmail.com. D0I:10.1523/JNEUROSCI.1106-08.2008

Copyright $\odot 2008$ Society for Neuroscience $\quad$ 0270-6474/08/2810751-15\$15.00/0 known about the neural mechanisms underlying internal model switching.

Recent studies have indicated that humans optimally determine their behaviors by combining predictions about environments based on previous knowledge with evidence from sensory feedback in a manner consistent with a Bayesian process (for review, see Körding and Wolpert, 2006). Empirically, two types of information are crucial for the switching of internal models. For example, when we lift an object, both contextual information, such as color or shape of the objects that can be perceived before movement execution, and error in the prediction of sensorimotor feedbacks that can be obtained during or after execution, contribute to the switching. Here, the error is a continuous value reflecting the difference between predicted and actual feedback (prediction error), which can be used to compute likelihood in a Bayesian process. A computational model for switching internal models, called the modular selection and identification for control (MOSAIC) (Wolpert and Kawato, 1998; Haruno et al., 2001) model, has proposed two separate switching architectures for each type of information.

In our previous behavioral study (Imamizu et al., 2007b), subjects learned to move their index fingers to targets while visual feedback of the finger movements was rotated clockwise (CW) or counterclockwise (CCW) by $40^{\circ}$ around the initial position. When subjects adapted to alternating blocks of opposing rotations, we investigated the effects of contextual information about 
forthcoming direction of the rotations on subjects' performances. Consequently, the contextual information selectively improved predictive switching performance, but did not affect switching performance based on prediction errors, suggesting the existence of two functionally independent mechanisms: a predictive mechanism based on contextual information and a postdictive mechanism based on prediction error.

In the current study, using functional magnetic resonance imaging ( $\mathrm{fMRI}$ ), we examined whether these two mechanisms are based on separate neural substrates. After training for the $40^{\circ}$ CW and CCW rotations, activity was measured while the direction of rotation was alternately changed. Subjects could predictively switch their behaviors based on previous contextual information in an instructed condition, but could not do so and relied on prediction errors in a noninstructed condition. We compared switching-related activity in the instructed condition to that in the noninstructed condition. Furthermore, using functional connectivity analysis, we investigated how information obtained by each mechanism is combined in the brain.

\section{Materials and Methods}

\section{Experimental design}

Experiments were designed so as to dissociate brain activity related to predictive switching based on contextual information from activity related to postdictive switching based on prediction error as follows.

Subjects moved their index fingers from the center start zone toward a target and back to the start zone (out-and-back pointing movement) in each trial while lying in the supine position (Fig. $1 A$ ). Subjects' forearms were fixed on a platform, and movements of the wrist and the metacarpophalangeal joint of the right index finger were allowed. Surgical tape fixed the distal and proximal interphalangeal joints of the finger. A marker of a position recording system (OPTOTRAK; Northern Digital) was attached to the right index fingertip. A cursor, targets, and the start zone were projected onto a screen, which was viewed by the subjects through a mirror. The cursor, corresponding to the position of the index finger tip, was always visible on the screen, and its position was determined by the marker position, which was projected onto a plane parallel to the screen $(x-y$ plane). A target set consisted of eight radially arrayed circles, separated in direction by $45^{\circ}$, placed on a circle with a radius of 5 $\mathrm{cm}$ on the screen (Fig. $1 \mathrm{~B}$, left panel), requiring a $10 \mathrm{~cm}$ movement of the marker for subjects to point at the targets. Only one of these targets appeared in each trial. The subjects were asked to start movements immediately after the target appeared, and to move in a fast and smooth motion without trajectory correction.

The cursor position was rotated $40^{\circ} \mathrm{CW}$ or CCW around the center of the screen as illustrated in Figure $1 C$. That is, when subjects moved their fingers, for example, in the directions indicated by the black arrows, the cursor on the screen moved in the directions indicated by the white arrows, depending on the direction of rotation. After intensive training with both CW and CCW rotations, brain activity was measured while the direction of rotation was alternately changed with pseudorandom timing. Subjects could predictively switch their behaviors under an instructed condition, in which the color of a cue as previous contextual information presented before the initiation of movement corresponded to the direction of rotation. However, under a noninstructed condition, in which the color did not correspond to the direction of rotation, subjects relied on prediction errors calculated from sensorimotor feedback.

Many previous studies have investigated the neural mechanisms involved when subjects switched from one arbitrary stimulus-response relationship (stimulus-response mapping) to another according to contextual information (Dove et al., 2000; Kimberg et al., 2000; Crone et al., 2006) (for review, see Murray et al., 2000; Passingham et al., 2000). For example, when the color of a contextual cue was green, subjects pressed a left key if a "+" sign was presented on a screen or a right key if a " - " sign was presented. However, when the color was red, the relationship between the sign and the key was reversed (Dove et al., 2000). This paradigm is different from that in the current study, because the task mainly

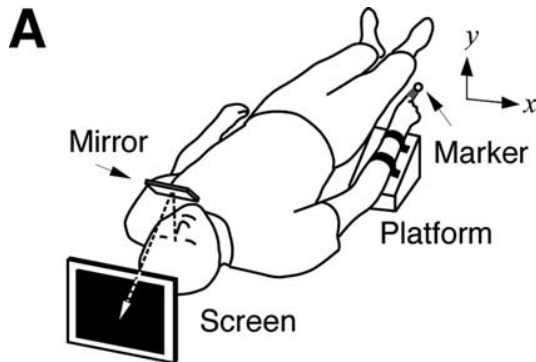

\section{B Screen}
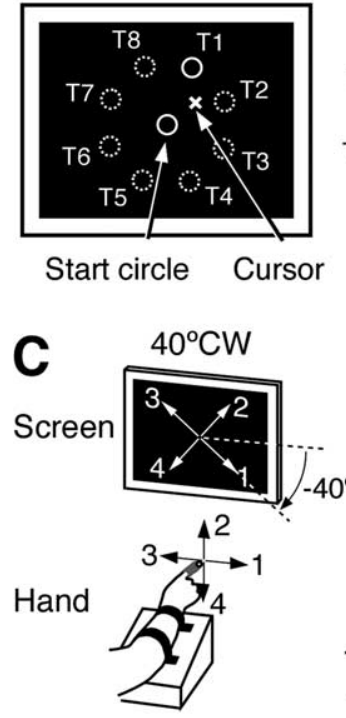

Figure 1. $A$, Posture of subjects during experiments both outside and inside the magnetic resonance (MR) scanner (scanner is not drawn in the figure). $\boldsymbol{B}$, Positions of the start circle and targets (T1-T8) on the screen (left panel) and in the hand space (right panel) under $40^{\circ}$ clockwise visuomotor rotation. " $E$ " indicates the angular error of a pointing movement. C, Relationship between the direction of finger movements (black arrows) and cursor movements (white arrows) under $40^{\circ} \mathrm{CW}$ (left panel) and $40^{\circ} \mathrm{CCW}$ (right panel) rotations. The numbers indicate correspondence between black and white arrows.

involves predictive switching of subjects' responses based on contextual information. Furthermore, switching is unlikely to be based on continuous prediction error between predicted and actual sensorimotor feedback, because subjects' responses are simple and stereotyped (namely, hand movement in the left or right direction). In contrast, the current experimental design included a noninstructed condition, in which predictive switching was not possible. Error in prediction of sensorimotor feedback is a continuous value in the above pointing movements, because the cursor can continuously move in any direction within the two-dimensional screen space and is not restricted in the two directions.

In our previous study (Imamizu et al., 2004) investigating regional differences between switching-related activity and internal-modelrelated activity, subjects manipulated three types of computer mouse with different input-output properties. Assuming that switching-related activity transiently increases after a change in mouse type, and that internal-model-related activity shows sustained activity specific to each mouse type, we investigated the temporal profiles of activity in various brain regions. Consequently, we identified switching-related activity mainly in the cerebellum, parietal regions, and Brodmann's area (BA) 46. However, the experimental design did not allow us to distinguish activity related to predictive switching from that related to postdictive switching for the following reasons. While subjects tracked a target continuously moving at high speed on a screen, the mouse type was changed, and, simultaneously, cognitive cues (change of cursor color and letters indicating the mouse type) were presented. In this way, subjects simultaneously obtained cognitive cues for predictive switching and sensorimo- 


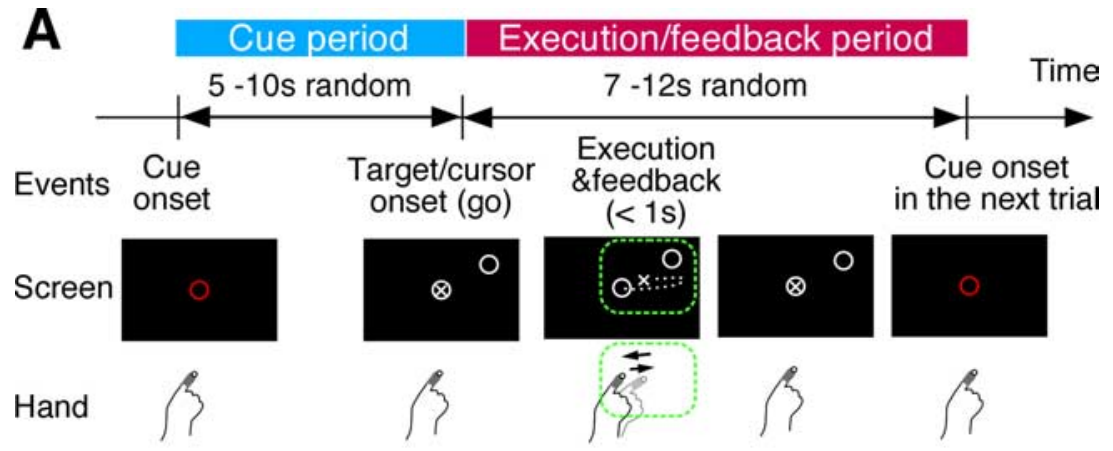

B

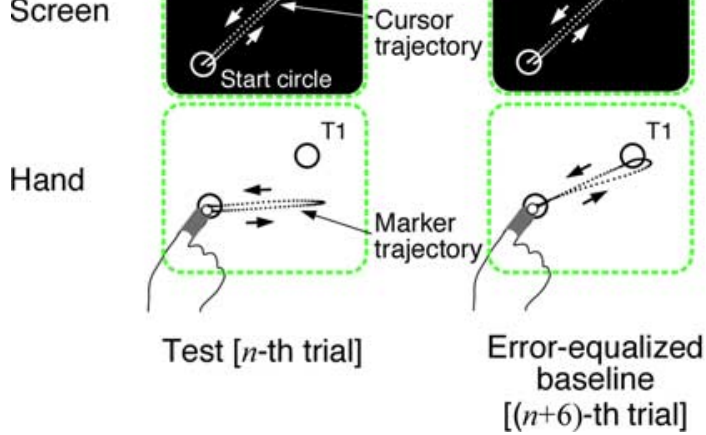

Error-zeroed and replayed

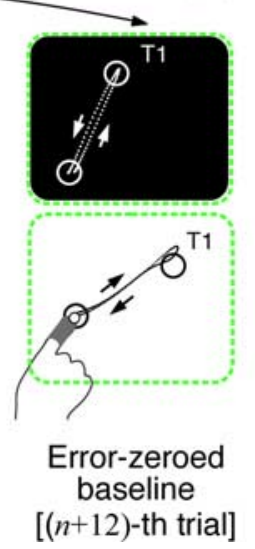

Figure 2. $\boldsymbol{A}$, Timeline in each trial of a switching session. Regions enclosed by green broken lines are magnified in $\boldsymbol{B}$. $\boldsymbol{B}$, Examples of cursor trajectories (top panels, white dots) and marker trajectories (bottom panels, black dots) from test (left), error-equalized baseline (middle), and error-zeroed baseline (right) trials. The dots indicate the positions of the cursor or the marker every $3 \mathrm{~ms}$. The arrows near the trajectories indicate moving direction.

tor feedback for postdictive switching; thus, cue-related activity temporally overlapped feedback-related activity. The current study used discrete pointing movements and event-related fMRI to separate activity related to presentation of the cognitive cue from that related to sensorimotor feedback.

\section{Subjects}

Fourteen subjects ( 10 males and four females; $19-44$ years of age) participated in this study. All participants were right-handed according to the Edinburgh Inventory (Oldfield, 1971). A signed informed consent form, approved by the institutional ethics committee, was obtained from each participant.

\section{Familiarization session outside the magnetic resonance scanner} Subjects conducted a familiarization session without the visuomotor rotations to become accustomed to pointing while lying in the supine position. The eight targets were presented randomly in each cycle of eight trials. One session consisted of 15 cycles (120 trials) and lasted 9 min.

\section{Training sessions outside the scanner}

Several days after the familiarization session, subjects were intensively trained to perform pointing movements with $40^{\circ} \mathrm{CW}$ and CCW rotation conditions. The targets and numbers of trials in each training session were the same as in the familiarization session. Subjects underwent 10 sessions with a 3 min break between each session. The direction of rotation changed alternately every session, and the order of rotations was counterbalanced between subjects. Before the onset of the first session, subjects were given the following instruction about the rotations while being shown Figure $1 \mathrm{C}$ : "The cursor position will be rotated $40^{\circ} \mathrm{CW}$ or CCW around the center of the screen. Thus, when you move your finger straight right, the cursor will move in a lower-right direction under the clockwise condition, but in an upper-right direction under the counter- clockwise condition. Similarly, when you move the finger straight up, the cursor will move in an upper-right direction under the clockwise condition, but in an upper-left direction under the counterclockwise condition, etc." During the break, subjects were informed of the rotation direction in the subsequent session.

\section{Switching sessions in the scanner}

A session consisted of 72 trials and lasted $\sim 20$ min. A total of 12 sessions were conducted on 4 or 5 separate days within a 2 week period. On each day, subjects underwent two or three sessions without getting out of the scanner. Subjects were repositioned in the scanner at the beginning of the first session of each day. A bite bar molded for each subject was attached to a fixed position of the head coil so that the subjects' heads were placed at the same position relative to the coil across different days. We carefully adjusted the slice positions of functional images so that the positions did not change across different days.

Each trial began with the presentation of a colored cue circle (diameter, $1.0 \mathrm{~cm}$ ) at the screen center (Fig. 2A). A white start circle replaced the cue circle after a random delay of 5-10 s, defined as the cue period. Simultaneously, a target appeared in the peripheral area of the screen, and the cursor appeared at the center of the start circle. Subjects were instructed to move the cursor to the target immediately on its appearance, and back to the start circle as soon as possible (within $1 \mathrm{~s}$ ), and then to maintain the cursor in the start circle. The cue circle for the next trial replaced the start circle after a random delay of 7-12 s from the target appearance, defined as an execution/ feedback period. The time intervals $(5-10$ or $7-12 \mathrm{~s}$ ) were inserted between the events because we used event-related fMRI to distinguish activity related to the cue and activity related to the execution/feedback. The cursor was always visible in the execution/feedback periods; thus, there was no time interval between execution and feedback, because such an interval might degrade subjects' performance and learning (Kitazawa et al., 1995). Therefore, instead of separating feedback-related activity of interest from execution/feedback-related activity using an event-related design, we measured execution-related activity during baseline periods and subtracted this activity from execution/feedback-related activity during the test periods (see below).

Within each session, a block was defined as a set of 18 trials. The direction of rotation did not change within a block, and whether or not it changed between successive blocks was randomly determined. Each block consisted of the following: (1) a test period; (2) an error-equalized baseline period; and (3) an error-zeroed baseline period in this order. Each period consisted of six trials, and six targets that were randomly selected from the eight possible targets (Fig. $1 B$ ) appeared in the sequence. The order of the six targets did not change across periods within a block; that is, the identical target appeared in the $n,(n+6)$, and $(n+$ 12)th trials. Sets of six targets were counterbalanced across blocks and sessions so that each target appeared an equal number of times over all sessions.

In a test period (Fig. $2 B$, left panel), subjects conducted pointing movements under $40^{\circ} \mathrm{CW}$ or CCW rotation conditions, and cursor trajectories were recorded in a computer memory. In the error-equalized baseline period (middle panel), the cursor moved toward a target according to the trajectory recorded in the preceding test period; that is, the cursor movement in the $(n-6)$ th trial was replayed on the screen during the execution/feedback period. Subjects were instructed to assume that the direction of rotation would be the same as that in the preceding test 
period, and to compensate for the rotation so as to move the cursor toward the target. The purpose of the error-equalized periods was to measure baseline activity related to visual stimuli (for example, target and motion of the cursor), movement execution, and visual feedback of performance error. In the error-zeroed baseline period (right panel), the task for subjects was the same as that in the error-equalized period. However, the cursor moved according to the recorded trajectory that was rotated around the center of the screen beforehand so that the cursor always moved to a target; that is, the pointing error is constantly zero. The purpose of the error-zeroed period was to measure baseline activity related to the visual stimuli and movement execution. Before the beginning of the experiment, subjects were informed of the above mechanisms of cursor movements in the error-equalized and the error-zeroed baseline periods.

The error-equalized baseline period, which occurred immediately after the test period, is more important than the error-zeroed baseline period for the following reasons. To identify switching-related brain regions, we looked for brain activity that increased immediately after alteration of rotation direction and decreased as the number of trials increased in the test periods (see below, Imaging data analysis). However, performance error is also supposed to increase after the alteration of rotation direction. It is known that this error evokes strong activity in large regions in the cerebral cortices and cerebellum (Imamizu et al., 2000; Diedrichsen et al., 2005). Thus, we measured baseline activity evoked by errors in the error-equalized period when switching was not needed and subtracted this activity from activity in the test period.

There were two conditions in the test period according to the existence of correspondence between the cue color and the direction of rotation. In the instructed condition, the color was red or blue when the rotation was $40^{\circ} \mathrm{CW}$ or CCW, respectively. Thus, subjects could know the direction of rotation in a cue period. However, in the noninstructed condition, the color was purple regardless of the rotation direction. In both conditions, the color was orange in the error-equalized baseline period and cyan in the error-zeroed baseline period.

\section{Magnetic resonance imaging acquisition}

A 1.5 T scanner (Shimadzu-Mariconi) was used to obtain blood oxygen level-dependent (BOLD) contrast functional images. Images weighted with the apparent transverse relaxation time were obtained with an echoplanar imaging sequence (repetition time, $3.5 \mathrm{~s}$; echo time, $50 \mathrm{~ms}$; flip angle, $90^{\circ}$ ). The whole brain was covered in 35 axial slices $(3 \mathrm{~mm}$ thickness; $1 \mathrm{~mm}$ gap), each of which was acquired as a $64 \times 64$ matrix (field of view, $192 \mathrm{~mm}$ ), with a voxel size of $3 \times 3 \times 4 \mathrm{~mm}$. Three hundred fifty-four volumes were acquired in each session. T1-weighted structural images were acquired with $1 \times 1 \times 1 \mathrm{~mm}$ resolution with a gradient echo sequence.

\section{Behavioral data analysis}

To gauge performance accuracy, we measured the angular error of the movement (Fig. 1B, right panel, "E"). The error was computed in the hand space as the absolute value of angle between the vector from the start circle to the target and the vector from the start circle to the marker position at the moment of first maximal velocity. We also investigated spatiotemporal data to examine whether subjects' performances differed among the above three periods. Movement initiation was defined as the first time when the tangential velocity of the marker crossed $5 \%$ of the maximum velocity of each trial. Movement termination was the last time the velocity fell $<5 \%$ of the maximum. We computed reaction time (interval between the target onset and the movement initiation), movement time (interval between the movement initiation and the termination), and the marker path length from movement initiation to its termination.

\section{Imaging data analysis}

Functional imaging data were analyzed using SPM2 (Wellcome Department of Cognitive Neurology, London, UK; http://www.fil.ion. ucl.ac.uk/spm). We discarded the first four volumes of images in each session to allow for $\mathrm{T} 1$ equilibration. For preprocessing, data were temporally realigned to correct for the sequence of slice acquisition and then spatially aligned to the first volume with a six-parameter rigid-body transformation. The data were spatially normalized to the Montreal Neurological Institute (MNI) (Montreal, Quebec, Canada) reference brain and resliced to a $2 \mathrm{~mm}$ isotropic voxel size. Data were smoothed spatially with a Gaussian kernel with $12 \mathrm{~mm}$ full-width at half-maximum. Because we were interested in differences in activity in global brain regions rather than local ones between predictive and postdictive mechanisms, a relatively large size filter was applied to ameliorate differences in intersubject localization. Data were high-pass filtered with a cutoff frequency of 1/128 $s$ to remove slowly varying trends.

To check the displacement of subjects' heads that might be caused by repositioning of subjects in the scanner at the beginning of each day after the second day (see above, Switching sessions in the scanner), we investigated the six-parameter values for spatial alignment of the first volume of images from each day with the first volume from the first day. Mean absolute values across days and subjects for translation $(x, y$, and $z)$ and rotation (pitch, roll, and yaw) were $1.15(\mathrm{SD}, 0.90) \mathrm{mm}, 1.30(0.96) \mathrm{mm}$, $1.01(0.91) \mathrm{mm}$, and $0.86^{\circ}\left(0.63^{\circ}\right), 0.64^{\circ}\left(0.45^{\circ}\right)$, and $0.96^{\circ}\left(0.76^{\circ}\right)$, respectively. Thus, the displacement was thought to be well within the range that can be corrected by SPM alignment.

General statistical analyses were performed in two stages. The firststage individual (fixed-effect) analysis was based on an event-related fMRI design. In this analysis, 72 types of events were defined: 2 (instructed and noninstruction conditions) $\times 3$ (test, error-equalized baseline and error-zeroed baseline periods) $\times 6(1-6$ th trials $) \times 2$ (cue and execution/feedback events). The cue or execution/feedback event was modeled by an impulse function locked at the onset of the cue or the execution/feedback period, respectively. The impulse functions were convolved with a canonical hemodynamic response function in SPM2 to yield regressors in a general linear model for each voxel as follows:

$$
S^{i}=\sum_{k=1}^{72} \beta_{k} x_{k}^{i}+\sum_{k=1}^{12} \gamma_{k} y_{k}^{i}+e .
$$

Here, $S^{i}$ is the fMRI activity in the $i$ th scan. $x$ s are regressors corresponding to the 72 types of events above explained. $y$ s are regressors of no interest corresponding to 12 sessions, each of which was assigned 1 in the corresponding session and 0 otherwise. Regression weights ( $\beta$ s and $\gamma \mathrm{s}$ ) and the residual $(e)$ were estimated for each voxel by the least-squares method. The estimated weights of interests $(\beta \mathrm{s})$ or contrasts of the weights were carried forward to a second-stage group (random effect) analysis using a one-way ANOVA model with nonsphericity correction and adjustment for correlated repeated measures. The results were inspected using $t$ contrasts according to the analysis designs explained below.

To average poststimulus BOLD responses for display purposes, we defined another model in the first-stage individual analysis using a finite impulse response (FIR) basis function with a bin width of $3.5 \mathrm{~s}$ (the repetition time of scans), modeling a total of six bins from 0 to $21 \mathrm{~s}$ after onset of one of the 72 aforementioned events. The model contains six regressors for an event of interest and regressors for the other events. Intuitively, the FIR basis set considers each time bin after stimulus onset to model the BOLD response that would have been seen in the absence of other events, and captures any possible shape of hemodynamic response function up to a given frequency limit. In this model, the estimated weights for each time bin are directly proportional to the average BOLD response at that time. This analysis was conducted by using a peristimulus histogram function in SPM2. We obtained the poststimulus response time courses for the events in the test trials and the error-equalized baseline trials. We subtracted the average response time course across the baseline trials from time course in each test trial, and displayed the subtracted time course averaged across subjects (see Fig. $6 B$ ).

(1) Analysis of activity related to cue presentation or execution/feedback of movements. To confirm whether the above event-related analysis could dissociate activity related to cue presentation from that related to execution/feedback of pointing movements, we applied the following analysis to the estimated $\beta$ weights for the first trials of test periods $\left(\beta_{\text {test }}^{1}\right)$, which are expected to be the most important trials for the switching of behaviors. We carried images of $\beta_{\text {test }}^{1}$ estimates in the first-stage analysis for 
four events (cue and execution/feedback events by instructed and noninstructed conditions) to the second-stage group analysis, and identified regions significantly activated by these events using a one-sample $t$ test $\left(\beta_{\text {teset }}^{1}>0\right)$ (see Fig. $\left.4 A, B, E, F\right)$. To confirm the subtraction of errorequalized baseline activity from the test activity, we made four contrast images in the first-stage analysis by subtracting the averaged estimate within the error-equalized baseline period $\left(\bar{\beta}_{\text {error-equalized }}\right)$, individually, from the estimates for the above four events $\left(C_{1}=\beta_{\text {test }}^{1}-\bar{\beta}_{\text {error-equalized }}\right)$. Then we carried them into the second-stage analysis to identify regions in which $C_{1}$ is significantly larger than zero (see Fig. $4 C, D, G, H$ ). The threshold in the second-stage analysis was $p<0.05$ when corrected for multiple comparisons at cluster level $(p<0.001$, uncorrected at voxel level and a cluster size $>270$ ). This threshold was low compared with those used in the imaging analyses described below $(2,4$, and 5), because we intended to confirm at this low threshold if our event-related design could dissociate activity related to cue presentation from that related to execution/feedback.

(2) Analysis of activity decreasing as a function of trial numbers after a change in rotation direction. According to behavioral data (described below), the size of angular errors increased immediately after a change in the direction of rotation and decreased gradually as a function of trial number. This suggests that adaptive switching processes proceeded gradually, and that activity directly related to these processes decreased gradually. To identify the activity related to switching, we conducted the following analysis. In the first-stage analysis, contrast images were generated by subtracting the averaged $\beta$ estimate within the error-equalized baseline period, individually, from the estimate for the cue or execution/ feedback event in the six test trials. In the second-stage analysis, a onesample $t$ test was applied to the contrast images to identify voxels in which the contrast of the $\beta$ estimates significantly decreased as a function of test trial number; that is, the weighted sum of the parameters $\left(5 \cdot C_{1}+\right.$ $3 \cdot C_{2}+1 \cdot C_{3}-1 \cdot C_{4}-3 \cdot C_{5}-5 \cdot C_{6}$ ) is significantly larger than zero. Here, $C_{i}$ indicates the contrast of the estimates obtained from the $i$ th test trial. The second-stage analysis was performed separately for four events (cue and execution/feedback events by instructed and noninstructed conditions). Decreasing activity evoked by cue events in the instructed condition and by execution/feedback events in the noninstructed condition are of particular interest, because the former activity is likely to be related to a predictive switch based on contextual information, and the latter activity is likely to be related to postdictive switching based on the error in prediction of sensorimotor feedback. The threshold was $p<0.05$ corrected at voxel level and a cluster size $>30$.

To investigate the actual time courses of the above decreasing activity across trials, we examined how the value of $C_{i}$ in individual subjects changed as a function of test trial numbers $(i)$ in regions identified by the second-stage analysis. $C_{i}$ is a contrast of $\beta$ estimates as described above. The magnitude of the estimate is proportional to the maximum height of the fitted hemodynamic response function and captures the size of the effect of the event on the hemodynamic response. Therefore, $C_{i}$ reflects the effect size in test trials in the absence of the effects that were measured in the error-equalized baseline trials. The $C_{i}$ value was divided by the mean signal intensity for a given voxel to normalize differences among voxels. We named this value "effect size." The effect size was averaged across subjects and voxels in a region of interest, and plotted as a function of test trial number (see Fig. 6C).

The above two types of decreasing activity were mainly observed in adjacent parietal regions [the superior parietal lobule (SPL), the inferior parietal lobule (IPL), and the precuneus]. To quantify the spatial segregation of the two types of activity, we measured the volume of the overlapping region in which both types of activity were observed, and compared it with the volume of regions in which either type was observed. For group volumetric analysis, we measured the volume of each type of activity detected and thresholded by the procedure described above. However, for individual analysis, because the above two types of activity were obtained by two-stage analysis [(1) subtraction of activity in the errorequalized baseline period from activity in the test periods in the individual-analysis stage, and (2) detection of a decreasing trend in activity with an increase in trial number by using the above contrast $\left(5 \cdot C_{1}\right.$ $+3 \cdot C_{2}+1 \cdot C_{3}-1 \cdot C_{4}-3 \cdot C_{5}-5 \cdot C_{6}$ ) in the group analysis stage], we could not conduct an individual analysis equivalent to the group analysis. Therefore, in individual volumetric analyses, we looked for activity evoked by cue or execution/feedback events in the first test trial, which is expected to be the most important trial for the switching of behaviors. Specifically, we subtracted averaged activity evoked by a cue event within the error-equalized baseline period from activity evoked by a cue event in the first test trial of the instructed condition, to extract activity related to a predictive switch. We subtracted averaged activity evoked by an execution/feedback event within the error-equalized baseline period from activity evoked by an execution/feedback event in the first test trial of the noninstructed condition to extract activity related to a postdictive switch. We applied a low threshold ( $p<0.001$, uncorrected) to each extracted activity to avoid underestimation of overlapping volume. We defined parietal regions of interest as the union of the SPL, IPL, and precuneus based on the Automated Anatomical Labeling map (Tzourio-Mazoyer et al., 2002) in the WFU PickAtlas toolbox (http://fmri.wfubmc.edu/cms/software) (Maldjian et al., 2003) for SPM2, and measured the volumes of regions related to either predictive or postdictive switches, and regions related to both.

(3) Analysis of changes in effective connectivity according to conditions. Dynamic causal modeling (DCM) is a system identification procedure that uses Bayesian estimation to make inferences about the effective connectivity between neural systems and how it is affected by experimental conditions (Friston et al., 2003). Because connectivity in DCM is measured through the coupling of changes in imaging signals, rather than anatomically, a significant unidirectional modularity influence of one brain region on another does not necessarily reflect the presence of a direct and unidirectional anatomical connection. Instead, the connectivity revealed by DCM reflects the inferred direction of neural influences that is specific to the experimental conditions, and that may be mediated through interneurons or brain regions not explicitly included in the model.

The results of the above analysis (2) suggested that activity in the SPL is related to predictive switching based on contextual information, and that activity mainly in the IPL and prefrontal cortex (PFC) is related to postdictive switching based on prediction error derived from sensorimotor feedback. We investigated how different conditions influenced effective connectivity between these regions and the lateral cerebellum, in which many studies have reported activity related to internal models in various kinds of sensorimotor tasks (Shidara et al., 1993; Shadmehr and Holcomb, 1997; Gomi et al., 1998; Kobayashi et al., 1998; Imamizu et al., 2000; Blakemore et al., 2001; Miall et al., 2001; Krakauer et al., 2004; Milner et al., 2007; Yamamoto et al., 2007). Only left cerebral hemisphere regions and right lateral cerebellar regions were included in our network to maintain model simplicity. Group maxima of activation were identified in the second-stage analysis described in (2). Then, subject-specific maxima were determined operationally as the nearest local maxima to the group maxima in regions significantly activated by any of the four events (namely, cue or execution/feedback events in the instructed or noninstructed conditions in the first test trial) in individual analyses ( $p<0.001$, uncorrected). Regarding the PFC, we found multiple activation clusters in BAs 10,11, and 46 in the second-stage group analysis, but selected the group maximum in BA 46 in which activity related to internal-model switching was also found in our previous study (Imamizu et al., 2004). Regarding the cerebellum, because subjects were required to compensate for the rotations in all trials in the current experiment, this design does not allow us to directly identify internal model activity based on a contrast between compensation and no-compensation (null) conditions. However, previous studies (Tamada et al., 1999; Imamizu et al., $2000,2004,2007 a$ ) have reported that internal models for the rotations are acquired near the posterior superior fissure. Thus, we anatomically located the group maximum around the center of the posterior superior fissure in the MNI reference brain $(x, y, z: 40,-60,-34)$. Because the lateral cerebellum was included in regions significantly activated by any of the above four events, we could identify subject-specific maxima in the cerebellum. Using SPM2, BOLD signal time courses were then extracted from $8 \mathrm{~mm}$ spheres centered on subject-specific maxima, and summarized as the principal eigenvariates of the time courses.

Effective connectivity analysis was performed using the DCM tool in 
SPM5. According to anatomical studies, mainly in monkeys, there exists connectivity between the cerebellum and parietal regions including the SPL and the IPL (Schmahmann and Pandya, 1989; Stein and Glickstein, 1992; Clower et al., 2001), between the cerebellum and the dorsolateral PFC (Kelly and Strick, 2003), and between the dorsolateral PFC and the parietal regions (Petrides and Pandya, 1999; Marconi et al., 2001). However, the exact connectivity among these regions in the human brain is unknown. Therefore, subject-specific DCMs were fully and reciprocally connected with modulatory influences from both conditions specified on all of the connections. The modulatory influences were modeled in the first test trial to maintain model simplicity. Input stimuli to the network were modeled as exerting direct effects on the SPL and the IPL. The effects of the input stimuli on the SPL model visual inputs evoked by cue presentation, and are exerted at the onsets of cue periods, whereas their effects on the IPL model sensorimotor-feedback inputs and are exerted at the onsets of execution/feedback periods.

The DCM analysis was performed in a twostage procedure similar to the functional image analysis explained above. The parameters corresponding to the strength of intrinsic or modularity effects obtained from the subjectspecific first-level DCM models were taken to a second between-subjects level analysis. A onesample $t$ test $(p<0.05)$ was used in the secondlevel analysis. Because it is unknown whether the DCM parameters were normally distributed, we confirmed that a nonparametric test (Wilcoxon's signed-rank test) yielded the same results as the $t$ test at the $p<0.05$ level. The threshold was not corrected for multiple comparisons because connections of interest were restricted by the results of the analysis (2).

(4) Analysis of increasing activity as switching process proceeds. To identify brain regions related to the degree of contribution of appropriate internal models, we looked for increasing activity as switching process proceeds. According to our previous behavioral and simulation study (Imamizu et al., 2007b), the time courses of signals reflecting the degree of contributions of appropriate internal models during the predictive switch based on contextual information were markedly different from the time courses during the postdictive switch based on prediction error. Specifically, the signal immediately increased after a change in the environment in the predictive switch condition, whereas it gradually increased with trial number in the postdictive switch condition. Based on this result, we looked for brain activity that increased immediately after a change in the direction of rotation in cue periods of the instructed condition, but gradually increased in execution/feedback periods of the noninstructed condition. Specifically, contrast images were yielded for each trial by the same subtraction as described in (2), in the first-stage analysis, and then a one-sample $t$ test was applied to the images to identify voxels in which the weighted sum of contrasts of $\beta$ estimates was significantly higher than zero. The weighted sum was defined as follows: $5 \cdot \mathrm{C}^{\text {cue }}{ }_{1}+$ $5 \cdot \mathrm{C}^{\text {cue }}{ }_{2}+5 \cdot \mathrm{C}^{\text {cue }}{ }_{3}+5 \cdot \mathrm{C}^{\text {cue }}{ }_{4}+5 \cdot \mathrm{C}^{\text {cue }}{ }_{5}+5 \cdot \mathrm{C}^{\text {cue }}{ }_{6}-15 \cdot \mathrm{C}^{\text {exe }}{ }_{1}-$ $11 \cdot \mathrm{C}_{2}^{\text {exe }}-7 \cdot \mathrm{C}^{\text {exe }}{ }_{3}-3 \cdot \mathrm{C}^{\text {exe }}{ }_{4}+1 \cdot \mathrm{C}^{\text {exe }}{ }_{5}+5 \cdot \mathrm{C}^{\text {exe }}{ }_{6}$. Here, $\mathrm{C}^{\text {cue }}{ }_{i}$ indicates a contrast of the estimates corresponding to the $i$ th trial in the cue period in the instructed condition (predictive switch). $\mathrm{C}^{\mathrm{exe}}{ }_{i}$ indicates a contrast corresponding to the $i$ th trial in the execution/feedback period in the noninstructed condition (postdictive switch). Weights for the $\mathrm{C}^{\text {cue }}{ }_{i}$ take the highest value (=5) from the first trial, and were constant across trials, but those for $\mathrm{C}^{\text {exe }}{ }_{i}$ gradually increased from the lowest ( $=-15$ in the first trial) to the highest $(=5$ in the last trial) values as trial number $(i)$ increased. These weights were adjusted so that their sum was zero. The threshold was $p<0.05$, corrected at voxel level and a cluster size $>30$ voxels. To investigate actual time courses of activity in regions of interest across trials, we plotted effect size [see above, (2)] calculated from the values of $\mathrm{C}^{\text {cue }}{ }_{i}$ and $\mathrm{C}^{\text {exe }}{ }_{i}$ as a function of test trial number (i).

(5) Analysis of activity related to visual feedback of performance error. We examined the difference between the error-equalized and the errorzeroed baseline periods to identify activity related to visual feedback of the error. Specifically, in the first-stage analysis, contrast images were yielded by subtracting $\beta$ estimates for the execution/feedback periods of the error-zeroed baseline trials from those for the execution/feedback periods of the error-equalized baseline trials. In the second-stage analysis, a one-sample $t$ test was applied to these images to identify voxels in which the contrast of the estimates was significantly higher than zero. The threshold was $p<0.05$ corrected at the voxel level and cluster size $>30$.

\section{Results}

\section{Behavioral data}

Figure $3 A$ shows subjects' performances in the familiarization and training sessions. The curves show angular error as a function of cycles (eight trials) in each session. The ordinate represents the average $( \pm S D)$ across subjects in which the value for each subject is the median size of errors within a cycle. The direction of rotation did not change within a training session, but alternately changed across the sessions. The error markedly increased at the beginning of each session, but gradually decreased as cycle number increased within the session. The error averaged within each session (horizontal lines) increased in the second training session, in which subjects experienced the opposing rotation for the first time, but decreased as the number of sessions increased. We applied a two-way (cycle by session) repeated-measures ANOVA to the medians of errors, and found significant effects of cycle $\left(F_{(14,182)}=2.71 ; p<0.0001\right)$ and session $\left(F_{(9,117)}=8.89 ; p<\right.$ $0.0001)$. According to Tukey's honestly significant difference 


\section{Activity evoked by cue presentation}

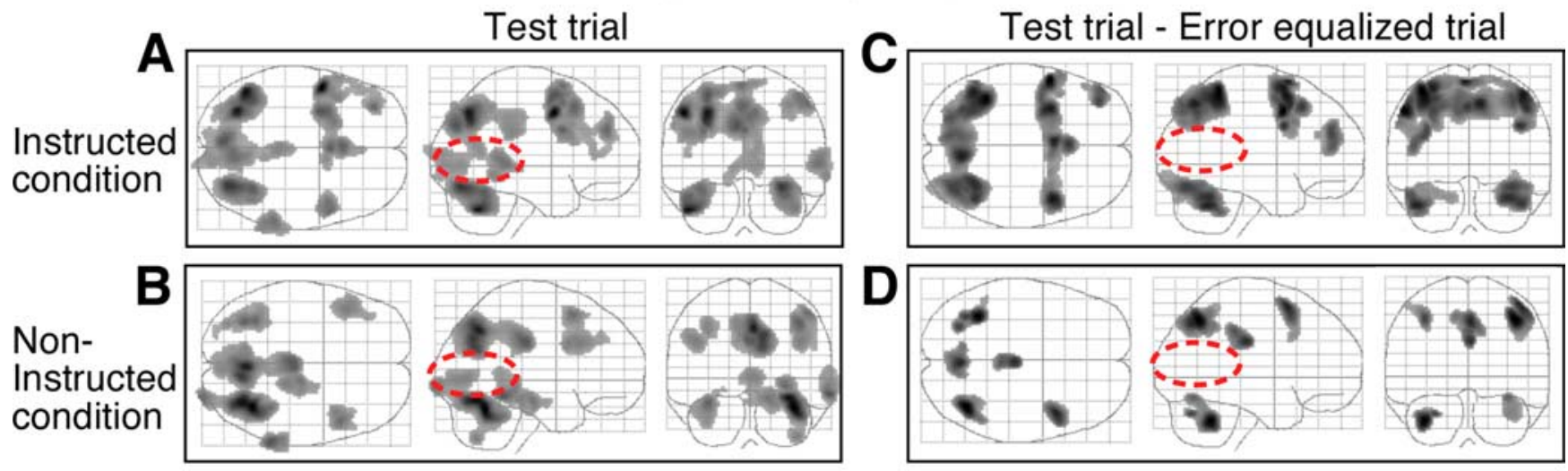

Activity evoked by execution/feedback of movement
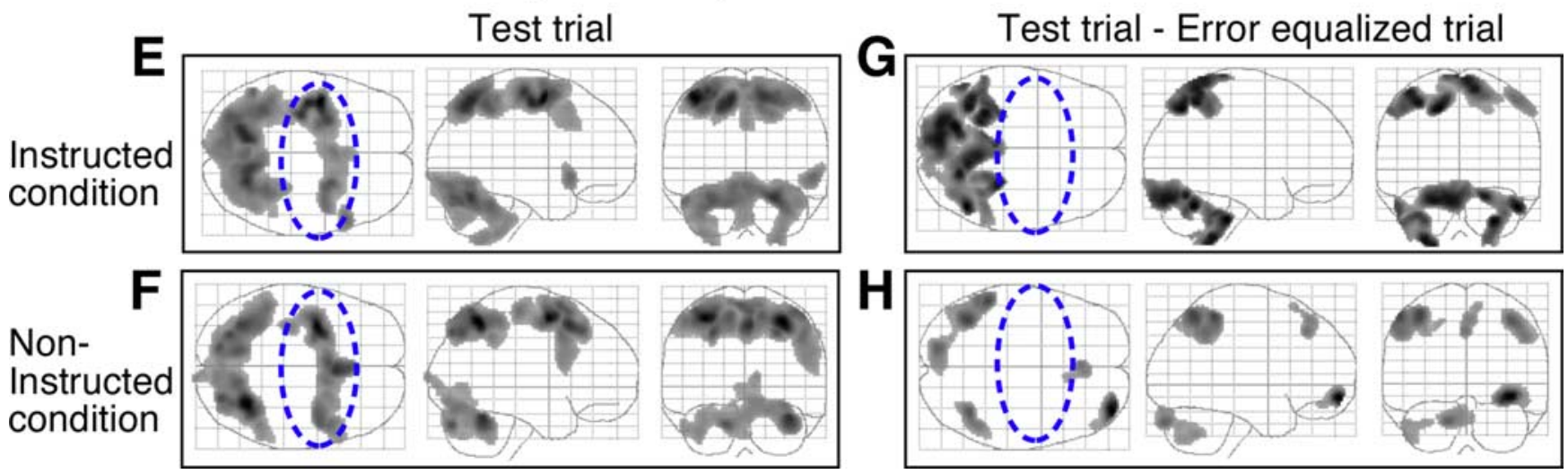

Figure 4. $\boldsymbol{A}-\boldsymbol{H}$, Activation evoked by cue presentation $(\boldsymbol{A}-\boldsymbol{D})$ or execution/feedback of movements $(\boldsymbol{E}-\boldsymbol{H})$ in between-subjects analysis. $\boldsymbol{A}, \boldsymbol{B}, \boldsymbol{E}$, and $\boldsymbol{F}$ show event-related activity in the first trial of test period without subtraction of activity in the error-equalized baseline period ( $p<0.05$, corrected for multiple comparisons at cluster level). $\boldsymbol{C}, \boldsymbol{D}, \boldsymbol{G}$, and $\boldsymbol{H}$ show activity in the first trial yielded by the contrast of the test period versus the error-equalized baseline period. The brain projections view the brain from above (transverse), right (sagittal), and behind (coronal). The red circles indicate the occipital regions related to visual information. The blue circles indicate regions mainly related to motor control (for example, the dorsal premotor regions, the supplementary motor regions, and the primary sensorimotor regions).

(HSD) post hoc test at $p<0.05$, the size of error averaged across sessions and subjects was smaller in the last cycle $\left(7.46^{\circ}\right)$ than the first cycle $\left(27.55^{\circ}\right)$, suggesting that error decreased within a session. Furthermore, error averaged across cycles and subjects was smaller in the last session than the second session, suggesting that it decreased across sessions after the second session. These results indicate short-term adaptation within each session as well as long-term learning of the opposing rotations across sessions after the second session. These results are consistent with our previous behavioral experiment investigating adaptation to opposing rotations, in which subjects were instructed about the direction of rotation when the rotation direction was changed (Imamizu et al., 2007b).

Figure $3 B$ shows subjects' performances in switching sessions in the scanner. The ordinate represents angular error averaged across sessions and subjects $( \pm S D)$. The abscissa represents trial number after a change in rotation direction. We computed, for each subject, condition, and trial number after the change, average angular error in the test period across sessions and applied a two-way (instructed or noninstructed condition by trial number after the change) repeated-measures ANOVA to the average error. The effect of trial number was significant $\left(F_{(5,65)}=88.73 ; p<\right.$ 0.0001 ), and the size of error was smaller in the sixth trial than in the first trial (Tukey's HSD post hoc test, $p<0.05$ ). An interaction between condition and trial number was significant $\left(F_{(5,65)}=\right.$
2.66; $p<0.03)$, suggesting a different condition effect that depended on the trials. When we compared the averaged errors between conditions for each trial, the error in the first trial was smaller in the instructed condition (mean $\pm \mathrm{SD}, 36.89 \pm 11.43$ ) than in the noninstructed condition $(46.27 \pm 12.64)$ (Tukey's HSD post hoc test, $p<0.05)$.

The error was almost constant in the error-equalized baseline period (Fig. 3B, middle) and the error-zeroed baseline period (right). We compared the error in the last trial of the test period as a reference to the errors in every trial of both baseline periods using Dunnett's test at the $p<0.05$ level, but could not find any significant difference. This suggests that the error in the baseline periods was maintained at the same level as the last trial of the test period.

Regarding subjects' performances in addition to error, we computed reaction time, movement time, and marker path length averaged across trials, sessions, and conditions for each subject and period. We applied one-way (period) repeatedmeasures ANOVA to the averaged values, and found no significant effect of period at $p<0.05$ level (reaction time, $F_{(2,39)}=$ 0.032 ; movement time, $F_{(2,39)}=0.27$; and path length, $F_{(2,39)}=$ $0.27)$. This result indicates that the subjects maintained constant performance throughout the test and the two baseline periods. The mean values across periods and subjects were $243.07 \mathrm{~ms}$ (SD, 
30.27) for reaction time, $244.17 \mathrm{~ms}$ (33.81) for movement time, and $22.25 \mathrm{~cm}(2.29)$ for out-and-back path length.

\section{Activity related to cue presentation or} execution/feedback of movements

Figure 4 shows group mean activity evoked by cue presentation (Fig. $4 A-D$ ) or execution/feedback of pointing movements (Fig. $4 E-H$ ). Figure $4, A, B, E$, and $F$, shows the evoked activity in the first trial of test period without subtraction of activity in the error-equalized baseline period [see Materials and Methods, (1)]. Figure 4, $C, D, G$, and $H$, shows activity in the first trial yielded by the contrast of the test period versus the error-equalized baseline period ( $p<0.05$, corrected for multiple comparisons at cluster level). Activity evoked by cue presentation was found bilaterally in prefrontal regions, dorsal premotor regions, parietal regions, occipital regions, and the lateral cerebellum, and in the left temporal regions (for detailed locations, see supplemental tables, available at www.jneurosci.org as supplemental material). These regions were activated in both the instructed and noninstructed conditions, but the activated regions were larger in the instructed condition (Fig. 4A) than in the noninstructed condition (Fig. $4 B)$. In the comparison of the test period versus the error-equalized baseline period (Fig. $4 C, D$ ), activation in the occipital regions related to visual information processing disappeared relative to the eventrelated activity without subtraction of activity in the error-equalized baseline period (red broken circles). Activity evoked by execution/feedback of pointing movements was found bilaterally in dorsal premotor regions, supplementary motor regions, intraparietal regions, and the lateral cerebellum, as well as in the left primary sensorimotor regions. These regions were activated in both the instructed and noninstructed conditions (Fig. $4 E, F$ ). In the comparison of test periods versus the error-equalized baseline period (Fig. $4 G, H)$, activation in regions mainly related to motor control, namely the dorsal premotor regions, the supplementary motor regions, and the primary sensorimotor regions, disappeared (blue broken circles), and restricted regions in the intraparietal regions and the lateral cerebellum remained. Almost identical activation patterns to those shown in Figure 4, C, D, G, and $H$, were obtained when we used data in the error-zeroed baseline period instead of the error-equalized baseline period.

In the baseline periods, subjects were instructed to assume that the same direc-
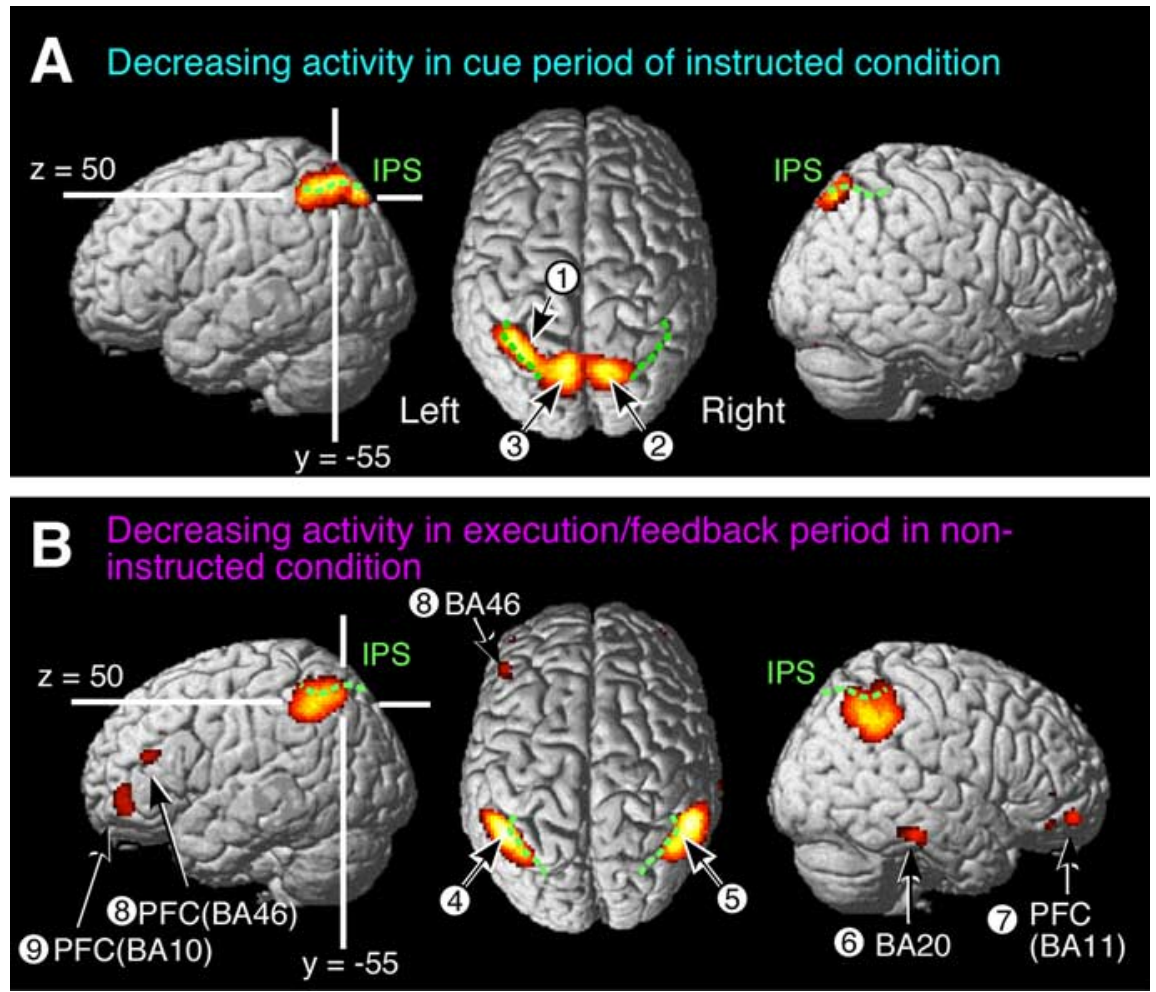

Figure 5. Activity decreases as trial number increases after switching in between-subjects analysis ( $p<0.05$, corrected for multiple comparisons at voxel level; cluster size, $>30$ voxels). $\boldsymbol{A}$, Decreasing activity in cue periods in the instructed condition. The broken green lines indicate the IPS. The left, middle, and right panels show the brain surface viewed from left, above, and right, respectively. The circled numbers indicate the local maxima of activation corresponding to the data in Table 1. $\boldsymbol{B}$, Decreasing activity in the execution/feedback period in the noninstructed condition.

Table 1. Summary of activations

\begin{tabular}{|c|c|c|c|c|c|}
\hline \multirow[b]{2}{*}{ Anatomical regions } & \multicolumn{3}{|c|}{ MNI coordinates } & \multirow[b]{2}{*}{ Cluster size } & \multirow[b]{2}{*}{$T$ value at peak } \\
\hline & $x$ & $y$ & $Z$ & & \\
\hline
\end{tabular}

Decreasing activity in the cue period in the instructed condition

SPL cluster

\begin{tabular}{|c|c|c|c|c|}
\hline (1) L superior parietal lobule & -32 & -60 & 54 & \\
\hline (2) R precuneus & 12 & -70 & 56 & 2335 \\
\hline (3) L precuneus & -8 & -68 & 54 & \\
\hline
\end{tabular}

Decreasing activity in the execution/feedback period in the noninstructed condition IPL

$\begin{array}{llllll}\text { (4) L inferior parietal lobule } & -48 & -52 & 50 & 918 & 7.08\end{array}$

$\begin{array}{lrrrrr}\text { (5) R inferior parietal lobule } & 46 & -60 & 50 & 1182 & 6.79\end{array}$

Temporal region

$\begin{array}{llllll}\text { (6) R inferior temporal gyrus (BA 20) } & 68 & -28 & -22 & 88\end{array}$

Prefrontal regions

$\begin{array}{lllll}\text { (7) R middle frontal gyrus (BA 11) } & 36 & 54 & -10 & 67\end{array}$

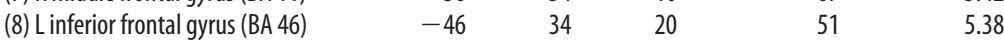

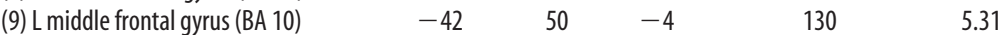

Activity that was high immediately after the switch in cue periods of the instructed condition and gradually increased in the execution/feedback periods in the noninstructed condition

$\begin{array}{lrrrrr}\text { (10) R LOTC } & 54 & -56 & -2 & 2354 & 7.91 \\ \text { (11) L LOTC } & -52 & -56 & 0 & 93 & 5.41 \\ \text { (12) R/L SMA/cingulate (BA 6) } & -6 & -4 & 48 & 1103 & 6.62 \\ \text { (13) L M1 (BA 4) } & -32 & -30 & 54 & 203 & 5.84 \\ \text { (14) L middle temporal gyrus (BA 21) } & -56 & -4 & -14 & 155 & 5.62 \\ \text { (15) R dorsal premotor region (BA 6) } & 52 & 2 & 48 & 86 & 5.27 \\ \text {-equalized baseline > error-zeroed baseline } & & & & & \\ \text { (16) R angular gyrus (BA 40) } & 54 & -50 & 32 & 8 & 10.10\end{array}$

Cluster size is represented by a number of $2 \mathrm{~mm}$ isotropic voxels. L, Left; $R$, right. 

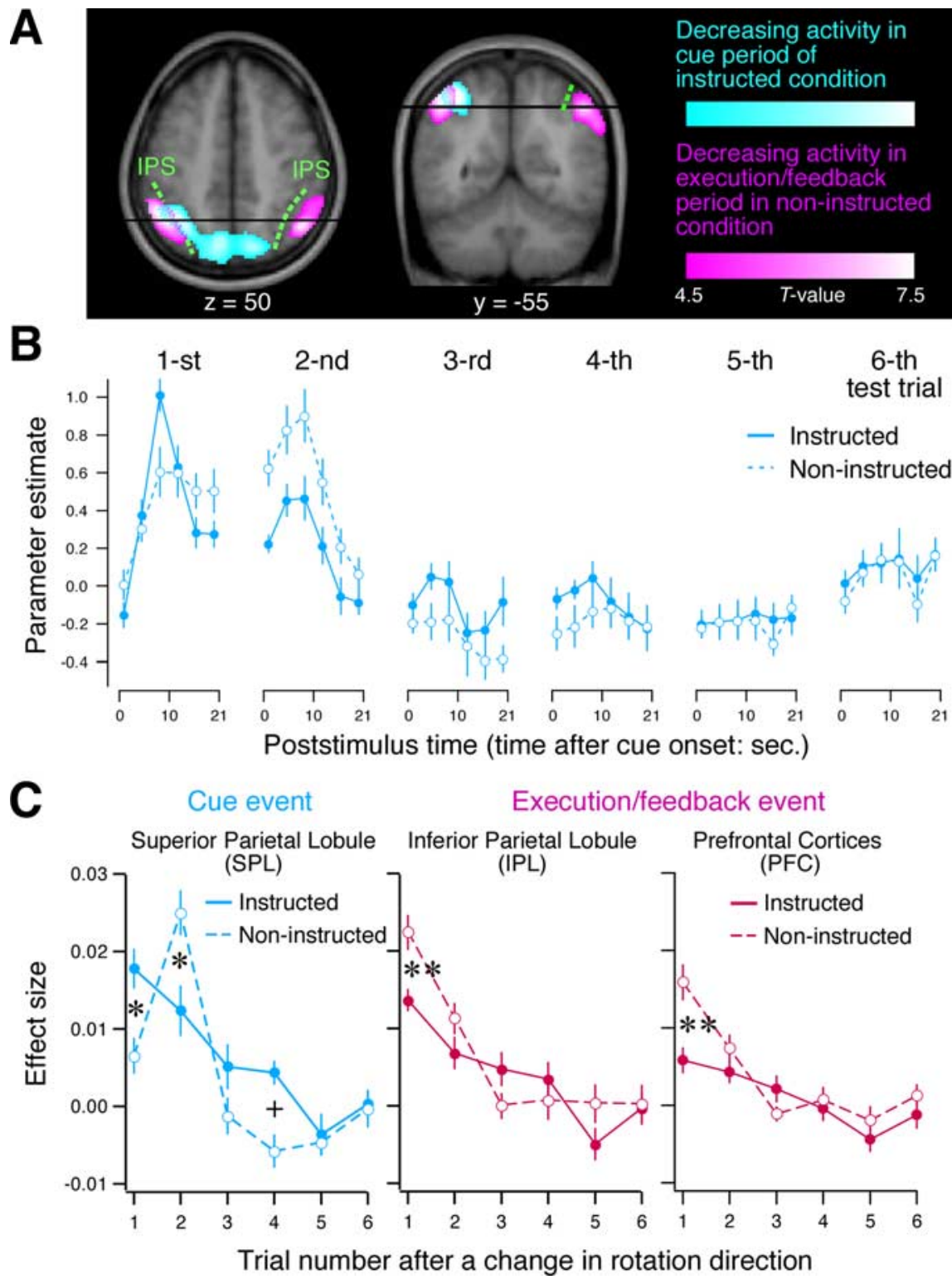

Figure 6. A, Spatial segregation of the two types of activity corresponding to Figure $5, A$ and $B$, around the IPS. Activations are superimposed on transverse (left) and coronal (right) slices from the subjects' averaged structural images at levels indicated by the white lines in the left panels of Figure $5, A$ and $B$. B , Poststimulus hemodynamic responses evoked by cue events in test trials after subtraction of responses in error-equalized baseline periods. The vertical axis represents values of parameter estimates (arbitrary units) from an FIR model that includes regressors modeling the response with a bin width of 3.5 s from 0 to 21 s after cue onset (see Materials and Methods). The response time courses were estimated for local maxima in the cyan regions of $A$ and averaged across the maxima and subjects ( \pm SE). $C$, Time courses for the sizes of the effects (arbitrary units) (see Materials and Methods) of cue or execution/feedback events on hemodynamic responses averaged across voxels and subjects ( $\pm \mathrm{SE}$ ) as a function of test trial number after a change in rotation direction. The left panel shows the size of the effect of the cue events in the cyan region of $\boldsymbol{A}$. The middle panel shows the size of the effect of the execution/feedback events in magenta regions. The right panel shows the size of the effect of the execution/feedback events averaged across PFC regions in Figure $5 B .{ }^{* *} p<0.001,{ }^{*} p<0.05,{ }^{+} p<0.10$, Tukey's post hoc test.

tion of rotation as in the preceding test period would be imposed, and to conduct pointing movements so as to compensate for the rotation. Thus, in the comparison of test periods versus the errorequalized baseline, the activation related to the compensation was subtracted, and the remaining activation was supposed to reflect information processing for the switching of internal models. As shown in Figure 3B, left panel, angular error after a change in rotation direction decreased as trial number increased, and reached an asymptotic level in the sixth trial, suggesting that switching is most needed in the first trial among the test periods and that the necessity for switching gradually decreased toward the sixth trial. Therefore, we looked for brain activity that decreased from the first to the sixth trial.

\section{Activity decreasing as a function of trial number after a change in rotation direction}

The activations shown in Figure 5 were identified to decrease in magnitude as the trial number increased in the group level analysis ( $p<0.05$, corrected for multiple comparisons; cluster size, $>30$ voxels) when contrast images in individual analyses were yielded by subtraction of activation in the error-equalized baseline periods from that in the test periods [see Materials and Methods, (2)]. Figure 5A shows decreasing activity in cue periods under the instructed condition, whereas Figure $5 B$ shows decreasing activity in the execution/feedback periods under the noninstructed condition. The locations of the activations are listed in Table 1 . The decreasing activity in the cue periods of the instructed condition constituted a large cluster in the bilateral SPL containing three activation peaks. The most anterior peak ( 1 in Fig. $5 A$, Table 1 ) existed in the medial wall of the left intraparietal sulcus (IPS). The other peaks existed in the precuneus of both hemispheres (2 and 3 ). In contrast, the decreasing activity in the execution/feedback periods of the noninstructed condition constituted large clusters in the bilateral IPL ( 4 and 5 in Fig. $5 B$, Table 1$)$ and relatively small clusters in the inferior temporal gyrus (6; BA 20) and prefrontal regions (7-9; BAs 11, 46, and 10). Regarding parietal activation, the decreasing activity in cue periods in the instructed condition (Fig. 6A, cyan region) existed medially to the IPS (dashed green line), whereas the decreasing activity in the execution/feedback periods of the noninstructed condition (magenta regions) existed laterally to the IPS. Almost identical activation patterns with those shown in Figures 5 and $6 A$ were obtained when we used data in the error-zeroed baseline period instead of the error-equalized baseline period.

The overlapping volume between the cyan and magenta regions in Figure $6 A$ was $2.1 \mathrm{~cm}^{3}$, whereas the volume of each region was $18.7 \mathrm{~cm}^{3}$ (cyan) or $16.8 \mathrm{~cm}^{3}$ (magenta). Thus, the overlap was only $6.3 \%$ of the union of the two regions. In individual volumetric analysis, we subtracted (1) activity evoked by cue events in the error-equalized baseline periods from activity evoked by cue events in the first test trial of the instructed condition, and (2) activity evoked by execution/feedback events in the error-equalized baseline periods from activity evoked by execu- 
tion/feedback events in the first test trial of the noninstructed condition $(p<0.001$, uncorrected). Then, we investigated the volumes of the two types of subtracted activity and the overlap between them in the parietal regions (see Materials and Methods). The overlapping volume averaged across subjects was 0.3 (SE, 0.1$) \mathrm{cm}^{3}$, whereas the volume of the activity related to predictive or postdictive switches was $5.1(\mathrm{SE}, 1.8)$ or $8.6(2.3) \mathrm{cm}^{3}$, respectively. Thus, the overlap was only $2.2 \%$ of the union volume of the two types of activity in the individual analysis.

Figure $6 B$ shows the time course of activity in the cue period of the test trials in the cyan regions of Figure $6 A$. Using the FIR model (see Materials and Methods), we estimated BOLD response time courses evoked by cue events in the test trials and the error-equalized baseline trials at local maxima in the cyan regions (Table 1, 1-3). We subtracted the average time course across the baseline trials from the time course in each test trial, separately for each maximum and subject. We plotted the subtracted time course averaged across the maxima and subjects ( \pm SE) as a function of poststimulus time in Figure $6 B$. Activation level gradually decreased as trial number increased in the instructed condition (solid lines). The level also decreased in the noninstructed condition (broken lines) across trials, but the level was highest in the second trial.

To quantitatively capture the above change in activity level across trials, we investigated the size of the effect of the cue event on hemodynamic responses in terms of the magnitude of the $\beta$ estimate in each test trial after subtraction of the estimate averaged across the error-equalized baseline trials [see Materials and Methods, (2)]. Figure 6C, left panel, shows the effect sizes of cue events averaged across voxels and subjects $( \pm S E)$ in the cyan regions of Figure $6 \mathrm{~A}$. The solid and broken lines correspond to the instructed and noninstructed conditions, respectively. We applied a two-way (condition by trial number) repeatedmeasures ANOVA to the effect size averaged across the voxels for each subject. An interaction between condition and trial number was significant $\left(F_{(5,65)}=13.92 ; p<0.0001\right)$, indicating a different condition effect that depended on the trials. Specifically, in the first trial, the effect size in the instructed condition was greater than that in the noninstructed condition (Tukey's post hoc test, $p<0.05)$. However, the opposite was true in the second trial, reversing again after the third trial.

Figure $6 C$, middle panel, indicates the time courses for the sizes of the effects of execution/feedback events across subjects and voxels in the magenta regions in Figure $6 A$. The effect sizes decreased as trial numbers increased in both the instructed and the noninstructed conditions. However, the interaction between condition and trial number was significant $\left(F_{(5,65)}=6.96 ; p<\right.$ $0.0001)$, and the effect size in the noninstructed condition was significantly greater than that in the instructed condition in the first trial (Tukey's post hoc test, $p<0.001$ ). Similar profiles were observed for the sizes of the effects of execution/feedback events averaged across subjects and voxels in three prefrontal clusters (BAs 11, 10, and 46) (Fig. 6C, right panel). We investigated the time courses for each prefrontal cluster, but could not find significant difference among the clusters.

\section{Change in effective connectivity according to conditions}

Figure $7 A$ shows subject-specific maxima of activations in the left SPL, the left IPL, the left PFC, and the right lateral cerebellum (CBL), from which BOLD signal time courses for the DCM analysis were extracted [see Materials and Methods, (3)]. Parameters corresponding to the magnitude of intrinsic influences (that is, connections that are independent of the condition) among these
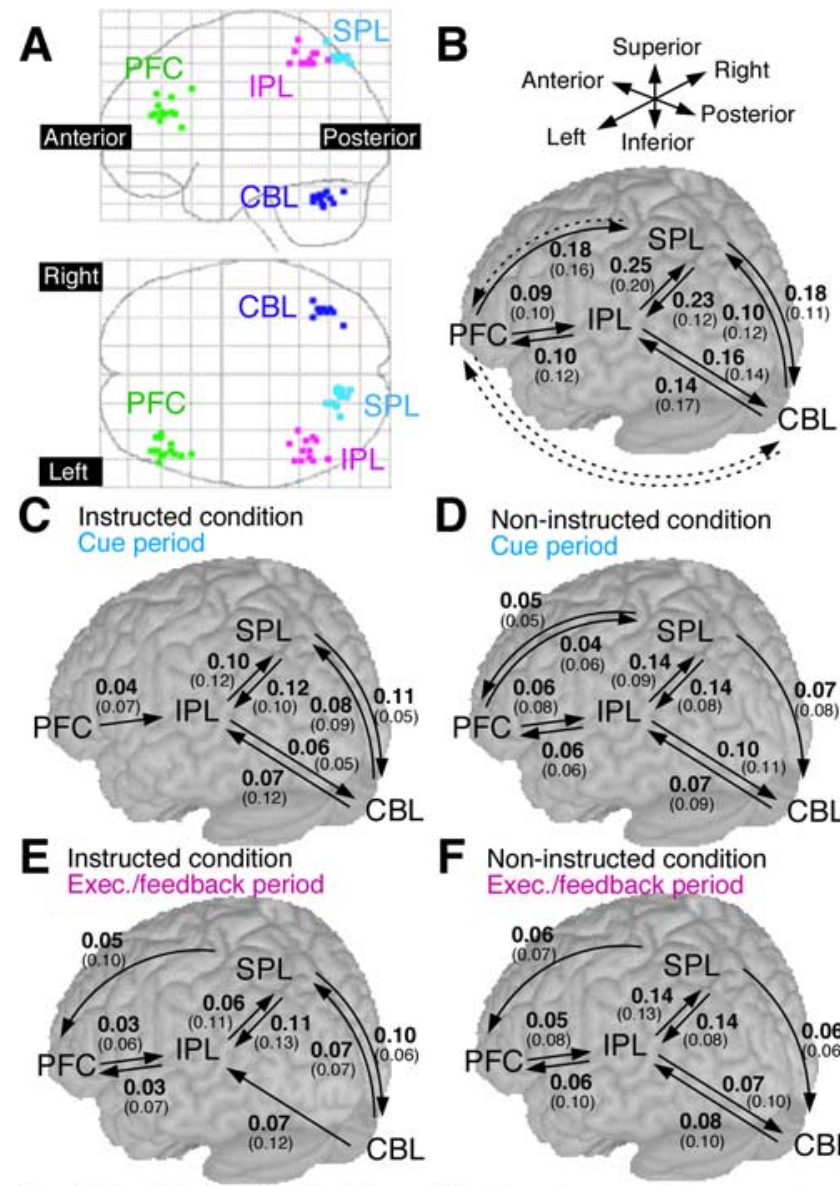

D Non-instructed condition

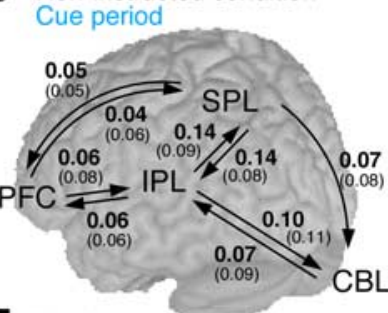

F Non-instructed condition
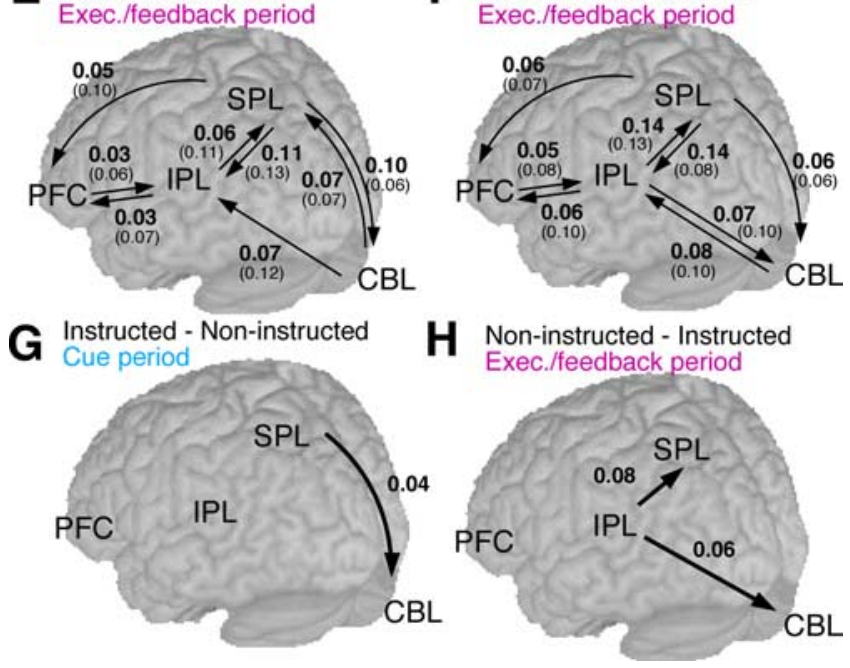

Figure 7. Results of DCM analysis. $\boldsymbol{A}$, Subject-specific maxima of activations from which BOLD signal time courses for DCM analysis were extracted. The brain projections view the brain from left (top) and above (bottom). $\boldsymbol{B}$, Intrinsic connections. The average parameters corresponding to strength of influences from a region to another across subjects (SD) are presented. The solid and dashed arrows indicate significant $(p<0.05)$ and nonsignificant influences, respectively. $\boldsymbol{C}-\boldsymbol{F}$, Modulation effects in the instructed $(\boldsymbol{C}, \boldsymbol{E})$ and noninstructed $(\boldsymbol{D}, \boldsymbol{F})$ conditions. Significant effects are presented in the cue $(\boldsymbol{C}, \boldsymbol{D})$ and execution/feedback periods $(\boldsymbol{E}, \boldsymbol{F})$. $\boldsymbol{G}$, Influences that are modulated by the instructed condition significantly more than by the noninstructed condition in the cue period. $\boldsymbol{H}$, Influences that are modulated by the noninstructed condition significantly more than by the instructed condition in the execution/feedback period.

regions were derived from the DCM analysis. Figure $7 B$ shows the means of the parameters across subjects. The solid arrows indicate parameters that were significantly larger than zero $(p<$ 0.05 ) in the between-subject level analysis. Figure 7, $C-F$, shows parameters corresponding to the magnitude of the modulation effect of the instructed (Fig. 7C,E) or noninstructed (Fig. 7D,F) conditions on the intrinsic influences in the first trial of the test period during the cue (Fig. 7C,D) or execution/feedback (Fig. $7 E, F)$ periods. As shown in these figures, a significant modulation effect of the conditions on the intrinsic influences could be identified at many connections. To identify an effect of contex- 
A

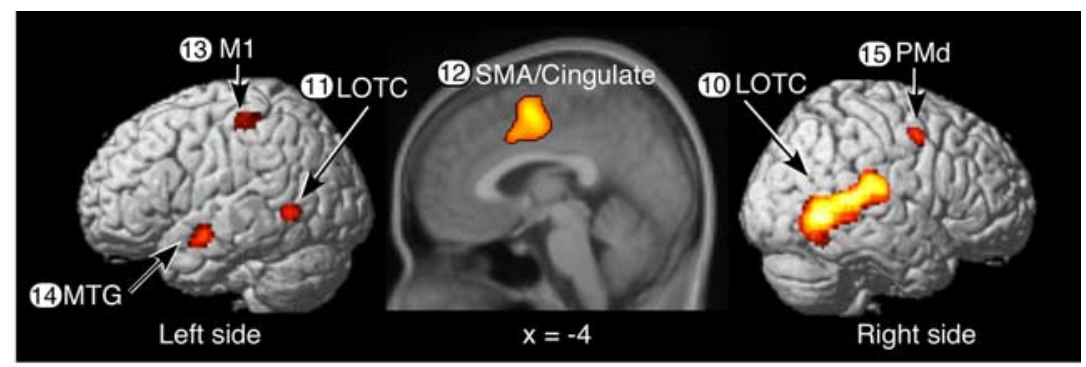

B

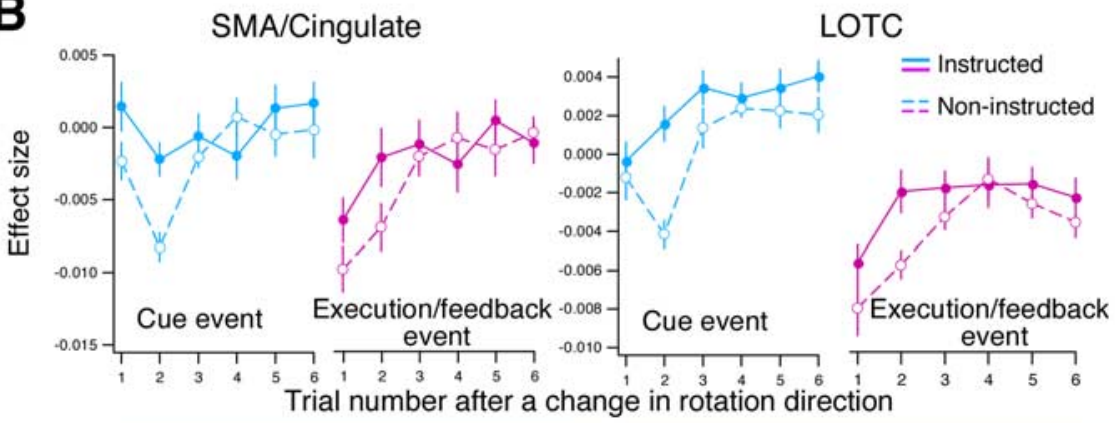

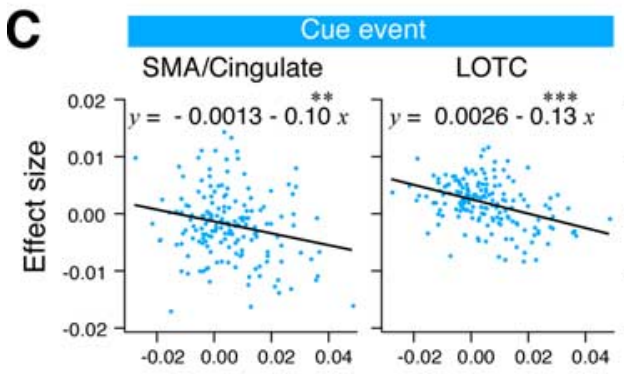

Effect size in SPL

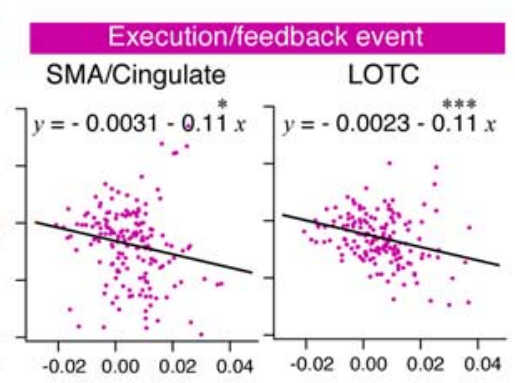

Effect size in IPL
Figure 8. A, Regions in which activation was high immediately after a change of rotation direction in cue periods in the instructed condition and gradually increased as trial number increased in execution/feedback periods in the noninstructed condition ( $p<0.05$, corrected at voxel level; cluster size, $>30$ voxels). The left and right panels show the brain surface viewed from the left and right, respectively. The middle panel shows activation superimposed on a midsagittal slice from the subjects' averaged structural images. $\boldsymbol{B}$, Time courses of activity measured as the size of the effect of cue (cyan) or execution/feedback (magenta) events on hemodynamic responses. Time courses were averaged across subjects and voxels in the SMA/cingulate (left panels) or LOTC (right panels) ( \pm SE), and plotted as a function of test trial number. The solid and dashed lines correspond to the instructed and noninstructed conditions, respectively. C, Correlations between the sizes of the effects of cue or execution/feedback events in parietal regions and each of the regions in $\boldsymbol{B}$. The left two panels show the correlation between the SPL and either the SMA/ cingulate or LOTC regarding the cue event. The right two panels show the correlation between the IPL and either the SMA/ cingulate or LOTC regarding the execution/feedback event. Each panel contains 168 points ( $=6$ test trials $\times 2$ conditions $\times 14$ subjects). The black lines were fitted to the points in each panel. ${ }^{* * *} p<0.001,{ }^{* *} p<0.01$, and ${ }^{*} p<0.05$ indicate significance levels in a one-sample $t$ test with the null hypothesis that the slope of the line would not be lower than zero (Bonferroni's correction for multiple comparisons).

tual information on switching, we examined each connection to determine whether the parameter values in the instructed conditions were higher than those in the noninstructed condition during the cue periods, and found a significant difference in influence from the SPL to the CBL between conditions (Fig. $7 G)(p<$ 0.05). Regarding an effect of sensorimotor feedback on switching, we examined whether the values in the noninstructed condition were higher than those in the instructed condition, and found a significant difference in influence from the IPL to the SPL and CBL between conditions (Fig. $7 H$ ).

\section{Increasing activity as switching process proceeds}

Figure $8 \mathrm{~A}$ shows the regions found by the analysis (4) (see Materials and Methods) to identify activation that was high immediately after a change in rotation direction in the cue periods in the instructed condition, and that gradually increased with trial number in the execution/feedback periods in the noninstructed condition. Large activation clusters were found in bilateral $\mathrm{SMA} /$ cingulate regions and the right lateral occipito-temporal cortices (LOTC). Figure $8 B$ shows time courses for effect size [see Materials and Methods, (2)] averaged across subjects and voxels in each region, separately for the cue and the execution/feedback events. Similar time courses were observed in both regions. We could confirm that the size of the effect of the cue event was high in the first trial in the instructed condition (cyan solid line), and that the size of the effect of the execution/ feedback event gradually increased in the noninstructed condition (magenta broken line). The size of the effect of the cue event in the noninstructed condition (cyan broken line) increased later than that in the instructed condition (cyan solid line). In contrast, the size of the effect of the execution/feedback event in the instructed condition (magenta solid line) increased earlier, namely, in the first and the second trials, than that in the noninstructed condition (magenta broken line). Similar time courses were observed in the left primary motor cortex (M1), the left middle temporal gyrus (MTG), and the right dorsal premotor regions (PMd) (Table 1).

The time courses shown in Figure $8 B$ are almost mirror patterns of those in Figure $6 C$ about a horizontal axis for corresponding event and condition, suggesting negative correlations. We investigated the relationships between the SPL and either the SMA or LOTC in terms of effect size after the cue event (Fig. 8C, left two panels), and those between the IPL and either the SMA or LOTC in terms of effect size after the execution/feedback event (right two panels). Each panel plots effect sizes (total 168 points) in test trials (6) combining data from the instructed and noninstructed conditions $(\times 2)$ and subjects $(\times 14)$. The slopes (regression coefficients) of lines fitted to the points were negative, as shown in the panels. Using a one-sample $t$ test, we tested the null hypothesis that the slope would not be lower than zero (a one-sided test). The hypothesis was rejected for every line $\left(t_{(166)}>2.33 ; p<0.05\right.$ after Bonferroni's correction for multiple comparisons), suggesting a negative correlation between the above regions in terms of their activity.

\section{Activity related to visual feedback of performance error}

Figure 9 shows the regions activated more in the error-equalized baseline period than in the error-zeroed baseline period. We could not identify an activation cluster exceeding 30 voxels, but found a small cluster (eight voxels) showing strong activation (peak $t$ value, 10.10) (Table 1) in BA 40 of the angular gyrus. In the above analyses, we found almost identical results when we used data from the error-zeroed baseline period instead of the 


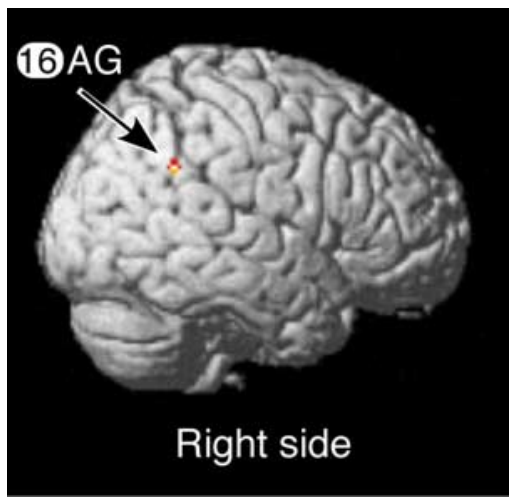

Figure 9. Regions activated significantly more in the error-equalized baseline period than the error-zeroed baseline period in the group analysis ( $p<0.05$, corrected at voxel level). $\mathrm{AG}$, Angular gyrus.

error-equalized baseline period. This is because the difference in activation between the two baselines was significant, but was restricted to a very small region in the angular gyrus.

\section{Discussion}

Activity in the SPL (Fig. 6A, cyan region) increased immediately after a change in the direction of rotation and decreased as trial number increased in the cue periods in the instructed condition. In the first test trial, the activity in this region, measured as the size of the effect of the cue event on the hemodynamic response in this region, was higher in the instructed condition than in the noninstructed condition (Fig. 6C, left panel), suggesting that this region is related to the predictive switch based on contextual information. Many studies have indicated contribution of the SPL to the planning of action based on visual information (Medendorp et al., 2005; Culham et al., 2006; Field et al., 2007). Kimberg et al. (2000) reported that the SPL is related to switching between different stimulus-response mappings based on contextual information. Our study suggests that the SPL associates contextual information with an appropriate set of relative contribution degrees of internal models for the current context.

It is known that SPL is activated by cues to shift spatial attention in a particular direction (Vandenberghe et al., 2001; Yantis et al., 2002). Our subjects could not know or predict a target position during the cue period as shown in Figure $2 A$. Thus, the SPL activity in the current experiment was unlikely directly related to a shift of attention. However, switching of behaviors often follows shifts of spatial attention; for example, switching between preparations for forehand and backhand strokes is determined according to spatial information about an approaching ball captured by attention systems. Therefore, shifts of spatial attention and predictive switching of behaviors and internal models are related to each other, and we speculate that they are based on neural mechanisms in the SPL regions. However, this may be dependent on the type of attention, because Pollmann et al. (2006) reported that the neural correlates of attentional shifts from one stimulus dimension to another are dissociated from those of behavioral changes.

Angular error after a change in rotation direction gradually decreased as trial number increased (Fig. $3 B$ ) even in the instructed condition, suggesting that the switching of internal models were not completed in the first trial. Therefore, switching was necessary in the subsequent trails, and both the switching mechanisms based on contextual information and the mechanism based on prediction error were activated. Consequently, the
SPL activity gradually decreased, even in the instructed condition, in proportion to the decrease in the angular error.

Activity in IPL and PFC regions (Figs. 5B; 6A, magenta region) decreased as trial number increased in the execution/feedback periods in the noninstructed condition. In the first test trial, the average effect size in these regions was higher in the noninstructed condition than in the instructed condition (Fig. 6C, middle and right panels), suggesting that this region is related to the postdictive switch. Grefkes et al. (2002) reported a contribution of the IPL to integration between visual and somatosensory information. In the current task, the IPL is expected to integrate visual and proprioceptive feedbacks, to compare them with predictions made by internal models and to calculate prediction error. We cannot directly measure the prediction error. However, it is likely to be equal to the angular error between the target direction and direction of the cursor movements because subjects were asked to move the cursor toward a target, and, thus, the prediction of the feedback is the cursor movement toward the target. The activity measured as the size of the effect of execution/ feedback events in the IPL (Fig. $6 C$, middle panel) decreased in proportion to the decrease in the angular error in the test period (Fig. 3B, left panel), suggesting that the IPL is related to prediction error. Our previous study (Imamizu et al., 2004) investigated activity related to the switching of internal models in a tracking task using a computer mouse, and found activation in the IPL and the PFC (BA 46). This suggests that the switching mechanism investigated in our previous study was mainly based on prediction error.

Diedrichsen et al. (2005) reported that activity related to errors under visuomotor rotation was spread over the precentral gyrus, the postcentral gyrus, the IPL, and the SPL. Because their task allowed corrective movements near a target, on-line correction of movement might evoke large activities. Our results suggest that, among the error-related activities in their study, the activity in the IPL is related to prediction error derived from sensorimotor feedback that contributes to the switching of internal models.

In the comparison between the error-equalized and the errorzeroed baseline periods, we found a small but strong activation in BA 40 (Fig. 9). Because switching of internal models was not needed in the error-equalized periods, errors contributing to switching activate large regions in the IPL, but errors that are unimportant for switching activate a small region in BA 40 .

Our connectivity analysis suggested that information flow changes according to the type of switching. The effect of conditions on the functional influence of the SPL on the lateral cerebellum was higher in the instructed condition than in the noninstructed condition (Fig. 7G). In light of previous studies suggesting that internal models are acquired in the cerebellum, top-down information from the SPL to the cerebellum contributes to predictive switching between internal models. In contrast, the effect of conditions on the influence of the IPL on the lateral cerebellum and the SPL increased more in the noninstructed condition than in the instructed condition (Fig. $7 H$ ). Information flow from the IPL to the cerebellum contributes to postdictive switching based on prediction error. The increased influence of the IPL on the SPL may reflect the flow of information about the rotation direction that was revealed by the prediction error and used as contextual information for the predictive switch in the subsequent trial. The SPL activity (Fig. 6C, left panel, dashed line) increased more in the cue event of the second trial in the noninstructed condition than in the first trial. The predictive switching mechanism could not work because the contextual in- 
A

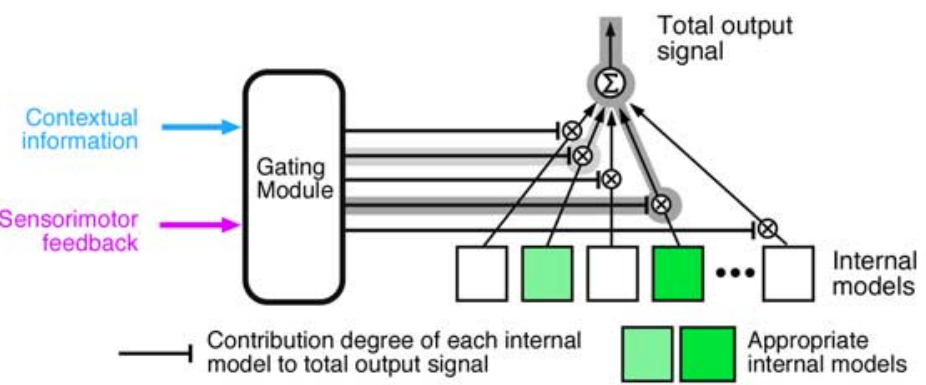

B

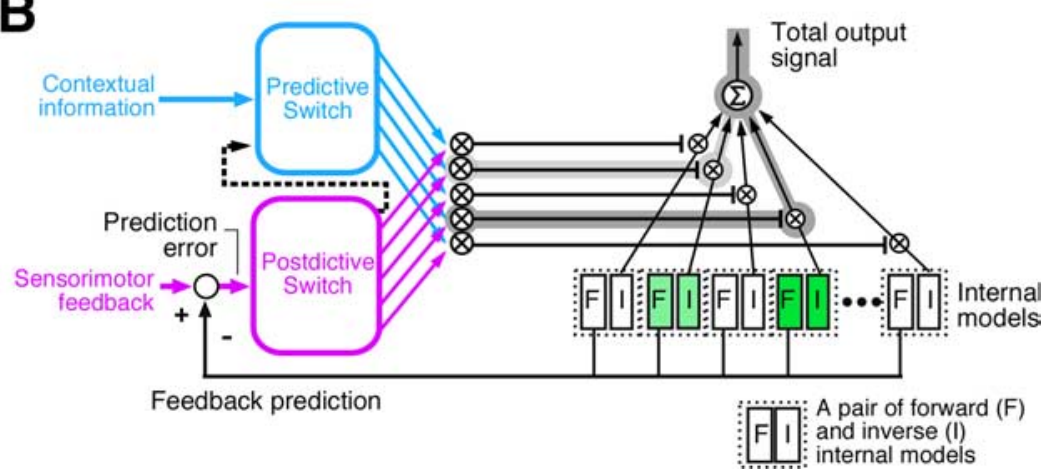

Figure 10. Computational models for the switching of internal models. $A$, Mixture-of-experts model having a single switching mechanism (a gating module). B, MOSAIC model having separate switching mechanisms for predictive switching based on contextual information and postdictive switching based on the prediction error of sensorimotor feedback. F, Forward internal models that predict sensorimotor feedback from an efference copy of a motor command. I, Inverse internal models that calculate motor commands from intended motion.

formation was not available in the first trial of the noninstructed condition, but a predictive switch is possible in the second trial if the mechanism can use rotation direction information generated by the IPL in the execution/feedback event of the first trial.

The mechanisms related to the increased influence from the IPL on the SPL may be analogous to those underlying behavioral adjustment after conflict or error in cognitive control tasks such as the Stroop color-naming task. Kerns et al. (2004) found that an increase in activity in the anterior cingulate cortex (ACC) in an error trial leads to an increase in activity in the PFC (BAs 8 and 9) in the subsequent trial, and suggested that the ACC monitors the conflict and that the PFC produces behavioral adjustments based on detection of the conflict. It can be postulated that the IPL is involved in the monitoring of error, and the SPL contributes to subsequent behavioral adjustment by predictive switching between internal models.

The activity in the PFC, including BA 46, increased in the first trial of the execution/feedback period in the noninstructed condition (Fig. $6 C$, right panel). Connectivity between the PFC and the parietal regions increased in the noninstructed condition (Fig. $7 D, F$ ) compared with the instructed condition (Fig. 7C,E), although the difference did not reach a statistically significant level. A possible reason for the PFC activity may be the execution of behavioral adjustment as suggested by the above study on cognitive control. Another reason specific to the current task is the reasoning of the rotation direction. Because subjects were informed of the two types of rotation, subjects could reason the current rotation direction based on the result of the first test trial. Goel and Dolan (2004) reported that BA 9, which exists in the dorsolateral PFC adjacent to BA 46, is involved in inductive reasoning. The activation of the dorsolateral PFC may reflect the inductive reasoning based on observation of results, and contributes to the switching in the next trial.
Decreasing activity in the execution/ feedback period in the noninstructed condition was found in BAs 10 and 11 (Fig. $5 B)$. These regions are lateral parts of the orbitofrontal cortex that receives projections from midbrain dopamine neurons and contribute to learning based on reward (for review, see Schultz, 2004). Successful switching with small performance error might be a type of reward for subjects and that reward learning mechanisms might be activated.

Figure $10 \mathrm{~A}$ illustrates the architecture of a mixture-of-experts model, which was proposed as a computational model for task switching. A single switching mechanism (a gating module) determines the degree of contribution of each internal model to the total output (Jacobs et al., 1991; Ghahramani and Wolpert, 1997). However, our current study suggested two separate mechanisms for predictive and postdictive switching, consistent with a recently proposed MOSAIC model (Wolpert and Kawato, 1998; Haruno et al., 2001) (Fig. $10 \mathrm{~B}$ ). The SPL is related to the predictive mechanism, whereas the IPL and the PFC are related to the postdictive mechanism. Output signals from the predictive mechanism correspond to an increase in the influence of the SPL on the cerebellum in the instructed condition (Fig. $7 G$ ), whereas output signals from the postdictive mechanism correspond to an increase in the influence of the IPL on the cerebellum in the noninstructed condition (Fig. $7 H)$. As discussed above, the influence of the IPL on the SPL in the noninstructed condition reflects the flow of information about the direction of rotation (Fig. $10 \mathrm{~B}$, dashed arrow) as revealed by the postdictive mechanism. The output signals from the two mechanisms are combined and determine the degree of contribution of the appropriate internal models to subject behaviors. The regions shown in Figure $8 A$ are related to the combined signals or the total output signal (Fig. $10 \mathrm{~B}$, gray regions) (for time courses of the signals, see supplemental figure, available at www. jneurosci.org as supplemental material).

Regarding the regions in Figure $8 \mathrm{~A}$, the LOTC (Bonda et al., 1996; Iacoboni et al., 2001; Schmid et al., 2001; Kawawaki et al., 2006) and the MTG (Rizzolatti et al., 1996) have been reported to contribute to the prediction and observation of movements. Output signals from forward internal models, which predict sensorimotor feedback from efference copy of motor command, play an important role in prediction and observation of movements of objects and other persons (Frith et al., 2000; Blakemore and Decety, 2001). Thus, the LOTC and MTG likely receive output signals from forward models. The other regions in Figure $8 \mathrm{~A}$ (SMA/ cingulate, M1, and PMd) are related to motor control, and likely receive output signals from inverse internal models, which calculate appropriate motor commands from intended motion. If the regions shown in Figure $8 \mathrm{~A}$ receive output signals from internal models, it is reasonable that activation of these regions increases with the number of trials after the change in rotation, and with the degree of contribution of appropriate internal models. 


\section{References}

Blakemore SJ, Decety J (2001) From the perception of action to the understanding of intention. Nat Rev Neurosci 2:561-567.

Blakemore SJ, Frith CD, Wolpert DM (2001) The cerebellum is involved in predicting the sensory consequences of action. Neuroreport 12:1879-1884.

Bonda E, Petrides M, Ostry D, Evans A (1996) Specific involvement of human parietal systems and the amygdala in the perception of biological motion. J Neurosci 16:3737-3744.

Clower DM, West RA, Lynch JC, Strick PL (2001) The inferior parietal lobule is the target of output from the superior colliculus, hippocampus, and cerebellum. J Neurosci 21:6283-6291.

Crone EA, Wendelken C, Donohue SE, Bunge SA (2006) Neural evidence for dissociable components of task-switching. Cereb Cortex 16:475-486.

Culham JC, Cavina-Pratesi C, Singhal A (2006) The role of parietal cortex in visuomotor control: what have we learned from neuroimaging? Neuropsychologia 44:2668-2684.

Diedrichsen J, Hashambhoy Y, Rane T, Shadmehr R (2005) Neural correlates of reach errors. J Neurosci 25:9919-9931.

Dove A, Pollmann S, Schubert T, Wiggings CJ, Cramon DY (2000) Prefrontal cortex activation in task switching: an event-related fMRI study. Cogn Brain Res 9:103-109.

Field DT, Wilkie RM, Wann JP (2007) Neural systems in the visual control of steering. J Neurosci 27:8002-8010.

Flanagan JR, Nakano E, Imamizu H, Osu R, Yoshioka T, Kawato M (1999) Composition and decomposition of internal models in motor learning under altered kinematic and dynamic environments. J Neurosci 19:RC34(1-5).

Friston KJ, Harrison L, Penny W (2003) Dynamic causal modelling. Neuroimage 19:1273-1302.

Frith CD, Blakemore SJ, Wolpert DM (2000) Abnormalities in the awareness and control of action. Philos Trans R Soc Lond B Biol Sci 355:1771-1788.

Ghahramani Z, Wolpert DM (1997) Modular decomposition in visuomotor learning. Nature 386:392-395.

Goel V, Dolan RJ (2004) Differential involvement of left prefrontal cortex in inductive and deductive reasoning. Cognition 93:B109-B121.

Gomi H, Shidara M, Takemura A, Inoue Y, Kawano K, Kawato M (1998) Temporal firing patterns of purkinje cells in the cerebellar ventral paraflocculus during ocular following responses in monkeys. I. Simple spikes. J Neurophysiol 80:818-831.

Grefkes C, Weiss PH, Zilles K, Fink GR (2002) Crossmodal processing of object features in human anterior intraparietal cortex: an fMRI study implies equivalencies between humans and monkeys. Neuron 35:173-184.

Gribble PL, Scott SH (2002) Overlap of internal models in motor cortex for mechanical loads during reaching. Nature 417:938-941.

Haruno M, Wolpert DM, Kawato M (2001) Mosaic model for sensorimotor learning and control. Neural Comput 13:2201-2220.

Iacoboni M, Koski LM, Brass M, Bekkering H, Woods RP, Dubeau MC, Mazziotta JC, Rizzolatti G (2001) Reafferent copies of imitated actions in the right superior temporal cortex. Proc Natl Acad Sci U S A 98:13995-13999.

Imamizu H, Miyauchi S, Tamada T, Sasaki Y, Takino R, Pütz B, Yoshioka T, Kawato M (2000) Human cerebellar activity reflecting an acquired internal model of a new tool. Nature 403:192-195.

Imamizu H, Kuroda T, Miyauchi S, Yoshioka T, Kawato M (2003) Modular organization of internal models of tools in the human cerebellum. Proc Natl Acad Sci U S A 100:5461-5466.

Imamizu H, Kuroda T, Yoshioka T, Kawato M (2004) Functional magnetic resonance imaging examination of two modular architectures for switching multiple internal models. J Neurosci 24:1173-1181.

Imamizu H, Higuchi S, Toda A, Kawato M (2007a) Reorganization of brain activity for multiple internal models after short but intensive training. Cortex 43:338-349.

Imamizu H, Sugimoto N, Osu R, Tsutsui K, Sugiyama K, Wada Y, Kawato M (2007b) Explicit contextual information selectively contributes to predictive switching of internal models. Exp Brain Res 181:395-408.

Jacobs RA, Jordan MI, Nowlan SJ, Hinton GE (1991) Adaptive mixture of local experts. Neural Comput 3:79-87.

Kawato M, Furukawa K, Suzuki R (1987) A hierarchical neural-network model for control and learning of voluntary movement. Biol Cybern 57:169-185.
Kawawaki D, Shibata T, Goda N, Doya K, Kawato M (2006) Anterior and superior lateral occipito-temporal cortex responsible for target motion prediction during overt and covert visual pursuit. Neurosci Res 54:112-123.

Kelly RM, Strick PL (2003) Cerebellar loops with motor cortex and prefrontal cortex of a nonhuman primate. J Neurosci 23:8432-8444.

Kerns JG, Cohen JD, MacDonald AW 3rd, Cho RY, Stenger VA, Carter CS (2004) Anterior cingulate conflict monitoring and adjustments in control. Science 303:1023-1026.

Kimberg DY, Aguirre GK, D’Esposito M (2000) Modulation of task-related neural activity in task-switching: an fMRI study. Brain Res Cogn Brain Res 10:189-196.

Kitazawa S, Kohno T, Uka T (1995) Effects of delayed visual information on the rate and amount of prism adaptation in the human. J Neurosci 15:7644-7652.

Kobayashi Y, Kawano K, Takemura A, Inoue Y, Kitama T, Gomi H, Kawato M (1998) Temporal firing patterns of purkinje cells in the cerebellar ventral paraflocculus during ocular following responses in monkeys. II. Complex spikes. J Neurophysiol 80:832-848.

Körding KP, Wolpert DM (2006) Bayesian decision theory in sensorimotor control. Trends Cogn Sci 10:319-326.

Krakauer JW, Ghilardi MF, Ghez C (1999) Independent learning of internal models for kinematic and dynamic control of reaching. Nat Neurosci 2:1026-1031.

Krakauer JW, Ghilardi MF, Mentis M, Barnes A, Veytsman M, Eidelberg D, Ghez C (2004) Differential cortical and subcortical activations in learning rotations and gains for reaching: a PET study. J Neurophysiol 91:924-933.

Kravitz JH, Yaffe FL (1972) Conditioned adaptation to prismatic displacement with a tone as the conditioal stimulus. Percept Psychophys 12:305-308.

Maldjian JA, Laurienti PJ, Kraft RA, Burdette JH (2003) An automated method for neuroanatomic and cytoarchitectonic atlas-based interrogation of fMRI data sets. Neuroimage 19:1233-1239.

Marconi B, Genovesio A, Battaglia-Mayer A, Ferraina S, Squatrito S, Molinari M, Lacquaniti F, Caminiti R (2001) Eye-hand coordination during reaching. I. Anatomical relationships between parietal and frontal cortex. Cereb Cortex 11:513-527.

Medendorp WP, Goltz HC, Crawford JD, Vilis T (2005) Integration of target and effector information in human posterior parietal cortex for the planning of action. J Neurophysiol 93:954-962.

Miall RC, Reckess GZ, Imamizu H (2001) The cerebellum coordinates eye and hand tracking movements. Nat Neurosci 4:638-644.

Milner TE, Franklin DW, Imamizu H, Kawato M (2007) Central control of grasp: manipulation of objects with complex and simple dynamics. Neuroimage 36:388-395.

Murray EA, Bussey TJ, Wise SP (2000) Role of prefrontal cortex in a network for arbitrary visuomotor mapping. Exp Brain Res 133:114-129.

Oldfield RC (1971) The assessment and analysis of handedness: the Edinburgh inventory. Neuropsychologia 9:97-113.

Osu R, Hirai S, Yoshioka T, Kawato M (2004) Random presentation enables subjects to adapt to two opposing forces on the hand. Nat Neurosci 7:111-112.

Passingham RE, Toni I, Rushworth MF (2000) Specialisation within the prefrontal cortex: the ventral prefrontal cortex and associative learning. Exp Brain Res 133:103-113.

Petrides M, Pandya DN (1999) Dorsolateral prefrontal cortex: comparative cytoarchitectonic analysis in the human and the macaque brain and corticocortical connection patterns. Eur J Neurosci 11:1011-1036.

Pollmann S, Weidner R, Müller HJ, Maertens M, von Cramon DY (2006) Selective and interactive neural correlates of visual dimension changes and response changes. Neuroimage 30:254-265.

Rizzolatti G, Fadiga L, Matelli M, Bettinardi V, Paulesu E, Perani D, Fazio F (1996) Localization of grasp representations in humans by PET: 1. Observation versus execution. Exp Brain Res 111:246-252.

Schmahmann JD, Pandya DN (1989) Anatomical investigation of projections to the basis pontis from posterior parietal association cortices in rhesus monkey. J Comp Neurol 289:53-73.

Schmid A, Rees G, Frith C, Barnes G (2001) An fMRI study of anticipation and learning of smooth pursuit eye movements in humans. Neuroreport 12:1409-1414. 
Schultz W (2004) Neural coding of basic reward terms of animal learning theory, game theory, microeconomics and behavioural ecology. Curr Opin Neurobiol 14:139-147.

Shadmehr R, Holcomb HH (1997) Neural correlates of motor memory consolidation. Science 277:821-825.

Shidara M, Kawano K, Gomi H, Kawato M (1993) Inverse-dynamics model eye movement control by Purkinje cells in the cerebellum. Nature 365:50-52.

Stein JF, Glickstein M (1992) Role of the cerebellum in visual guidance of movement. Physiol Rev 72:967-1017.

Tamada T, Miyauchi S, Imamizu H, Yoshioka T, Kawato M (1999) Cerebro-cerebellar functional connectivity revealed by the laterality index in tool-use learning. Neuroreport 10:325-331.

Tzourio-Mazoyer N, Landeau B, Papathanassiou D, Crivello F, Etard O, Delcroix N, Mazoyer B, Joliot M (2002) Automated anatomical labeling of activations in SPM using a macroscopic anatomical parcellation of the MNI MRI single-subject brain. Neuroimage 15:273-289.
Vandenberghe R, Gitelman DR, Parrish TB, Mesulam MM (2001) Functional specificity of superior parietal mediation of spatial shifting. Neuroimage 14:661-673.

Wada Y, Kawabata Y, Kotosaka S, Yamamoto K, Kitazawa S, Kawato M (2003) Acquisition and contextual switching of multiple internal models for different viscous force fields. Neurosci Res 46:319-331.

Wolpert DM, Kawato M (1998) Multiple paired forward and inverse models for motor control. Neural Netw 11:1317-1329.

Wolpert DM, Ghahramani Z, Jordan MI (1995) An internal model for sensorimotor integration. Science 269:1880-1882.

Yamamoto K, Kawato M, Kotosaka S, Kitazawa S (2007) Encoding of movement dynamics by Purkinje cell simple spike activity during fast arm movements under resistive and assistive force fields. J Neurophysiol 97:1588-1599.

Yantis S, Schwarzbach J, Serences JT, Carlson RL, Steinmetz MA, Pekar JJ, Courtney SM (2002) Transient neural activity in human parietal cortex during spatial attention shifts. Nat Neurosci 5:995-1002. 